\title{
Interpretation of potential intermittence titration technique experiments for various Li-intercalation electrodes*
}

\author{
M.D.Levi ${ }^{1}$, D.Aurbach ${ }^{1}$, M.A. Vorotyntsev ${ }^{2}$ \\ 1 Department of Chemistry, Bar-Ilan University, Ramat-Gan 52900, Israel \\ 2 LSEO, Faculté des Sciences Gabriel, Université de Bourgogne, \\ 6 boulevard Gabriel, 21000 Dijon, France
}

Received November 15, 2001

\begin{abstract}
In this paper we compare two different approaches for the calculation of the enhancement factor $W_{i}$, based on its definition as the ratio of the chemical and the component diffusion coefficients for species in mixed-conduction electrodes, originated from the "dilute solution" or "lattice gas" models for the ion system. The former approach is only applicable for small changes of the ion concentration while the latter allows one to consider a broad range of intercalation levels. The component diffusion coefficient of lithium ions has been determined for a series of lithium intercalation anodes and cathodes. A new "enhancement factor" for the ion transport has been defined and its relations to the intercalation capacitance and the intercalation isotherm have been established. A correlation between the dependences of the differential capacitance and the partial ion conductivity on the potential has been observed. It is considered as a prove that the intercalation process is controlled by the availability of sites for Li-ion insertion rather than by the concurrent insertion of the counter-balancing electronic species.
\end{abstract}

Key words: Li-ion battery, binary component, chemical diffusion coefficient, ionic conductivity, lattice gas model

PACS: 84.60.Dn, 66.30.Dn

\section{Introduction}

Thermodynamic and kinetic behaviour of various mixed-conduction solids, in particular Li-alloys (e.g. $\mathrm{Li}_{X} \mathrm{Al}$ or $\mathrm{Li}_{1+Y} \mathrm{Sb}$ ) have been carefully studied both theoretically and experimentally in the late $70^{\text {ties }}$ by Weppner and Huggins [1-3]. Their approach was based on the so-called "dilute solution" approximation, for which the enhancement factor $W_{i}$ was defined as the ratio of the chemical diffusion coefficient of ions, $D_{e i}$, and the component diffusion coefficient $D_{i}$. The total variation of $X$ with

*This paper is dedicated to Jean-Pierre Badiali on the occasion of his 60th birthday 
potential was considered to be small ( $X \ll 1$ ), thus the partial ionic conductivity $\kappa_{i}$ for mixed-conduction electrode, $\kappa_{i}$, is parameterized within the "dilute solution" approach by a quantity proportional to the product of the ion electric mobility (and, hence the component diffusion coefficient) and the bulk ion concentration.

An alternative approach which may be related to an intercalation electrode with large variation in $X$ from 0 to 1 , has been advanced by Chidsey and Murray [4]. They considered the redox-capacity of electroactive polymer films, $C_{\text {int }}$ being limited by availability of sites for insertion of electrons in the bulk film. Therefore, in their treatment the kinetic properties of these mixed-valence electrodes were associated primarily with electron properties, for which a more realistic description (compared to "the dilute solution" approximation [1]) has been proposed in the framework of a simple lattice-gas model [5]. The direct current $(d c)$ electron conductivity, $\kappa_{e}$, was represented as the product of the redox-capacity $C_{\text {int }}$ and the chemical diffusion coefficient for electrons, $D_{e}[4]$. Therefore, $D_{e}$ could be easily calculated as a function of potential using a combination of $d c$ conductivity measurements and linear scan voltammetry $\left(C_{\text {int }}\right.$ is proportional to the measured voltammetric current for sufficiently slow scan rates).

In contrast to the case of electroactive polymers, for Li-intercalated compounds it is often the availability of sites in the host's bulk that limits the intercalation capacity, as was suggested by McKinnon [6]. Although the partial ionic conductivity of Li-insertion compounds can, in principle, be measured by $d c$ current technique, as was described by Weppner and Huggins for Li-alloys [3], a lot of experimental difficulties may complicate the measurements due to the fact that practical Li-ion insertion electrodes are mostly powdery porous composites. On the other hand, the partial ionic conductivity $\kappa_{i}$ as a function of potential can be measured with a good accuracy using one of the appropriate relaxation techniques such as potentiostatic or galvanostatic intermittent titration techniques (PITT and GITT, respectively), or electrochemical impedance spectroscopy (EIS), similar to that described for the titration of Li-alloys $[2,7]$.

Our first goal is to perform an adequate parameterization of this quantity, $\kappa_{i}$, similar to the parameterization of $\kappa_{e}$ described above [4], with further comparison between the experimental $\kappa_{i}$ vs. $E$ curves and the theoretical ones obtained in the framework of a simple lattice-gas model. Such comparison will allow us to propose a new method for the reliable determination of the new component diffusion coefficient of ions, $D_{i}^{\circ}$. When contrasting this value with the plots of the chemical diffusion coefficient of Li-ions vs. potential, potential dependence of the ratio $W_{i}^{\circ}=D_{e i} / D_{i}^{\circ}$, defined as a new "enhancement factor", can be attributed to the interactions between the intercalation sites, either attractive or repulsive. Thus, the physical factors manifesting themselves in the potential dependences of $W_{i}$ and $W_{i}^{\circ}$ are different. Our second goal will be to correlate the classical "enhancement factor" $W_{i}$ with an analytical expression for the related intercalation isotherm. Such a correlation can be elucidated taking into consideration the stoichiometry of the bulk association-dissociation reaction between the electronic and ionic species in the mixed-conduction electrode. 


\section{Experimental}

The mode of preparation of vacuum-deposited $\mathrm{V}_{2} \mathrm{O}_{5}$ films further heat-treated at $350{ }^{\circ} \mathrm{C}$ for better crystallization was previously reported $[8,9]$. Here we worked with $\mathrm{V}_{2} \mathrm{O}_{5}$ films $1600 \AA$ thick. All other 6 electrodes were composites consisting of the related powdery mass mixed with polyvinylidene fluoride (PVdF) binder and electrically conductive carbon black (only in the case of the cathodes). Details can be found in previous publications related to graphite [10,11], disordered carbons [12], $\mathrm{LiCoO}_{2}$ [13], $\mathrm{LiNiO}_{2}$ and $\mathrm{LiNi}_{0.8} \mathrm{Co}_{0.2} \mathrm{O}_{2}$ [14,15]. In brief, graphite electrodes were composed of $90 \%$ of synthetic graphite powder KS 6 (Lonza, $6 \mu \mathrm{m}$ average particle size along the basal plane, $0.1-0.5 \mu \mathrm{m}$ thick) and $10 \% \mathrm{PVdF}$ binder. A piece of $\mathrm{Ni}$ or $\mathrm{Cu}$ foil $(1.2 \times 1.2 \mathrm{~cm})$ served as a current collector. Composite disordered carbon electrodes contained $90 \%$ of disordered carbon produced by Mitsubishi $(10 \mu \mathrm{m}$ average particle size) and $10 \% \mathrm{PVdF}$ binder. The technique of electrode preparation was identical to that of graphite electrode.

$\mathrm{LiCoO}_{2}, \mathrm{LiMn}_{2} \mathrm{O}_{4}, \mathrm{LiNiO}_{2}$ and $\mathrm{LiNi}_{0.8} \mathrm{Co}_{0.2} \mathrm{O}_{2}$ powders were obtained from Merck (2-3 and $0.5-1 \mu \mathrm{m}$ particle size, for the former and three latter powders, respectively). The electrode's active mass was $85 \mathrm{wt} \%$ of one of the above transition metal oxides, $10 \mathrm{wt} \%$ conductive carbon black, and $5 \mathrm{wt} \% \mathrm{PVdF}$ binder. These substances were thoroughly mixed with an appropriate amount of 1-methyl-2-pyrrolidone, with subsequent sonication in a test tube. Using a micropipette, several drops of this suspension (under continuous sonication) were removed from the middle level of the tube, then uniformly spread on one side of a heated $1.2 \times 1.2 \mathrm{~cm}$ piece of $\mathrm{Al}$ foil. After drying in an oven at $150{ }^{\circ} \mathrm{C}$, the other side of the foil was similarly covered with the same active mass. Each electrode contained about 1-2 mg of the active mass (a few microns thick).

A three-electrode cell contained a polyethylene frame with symmetrical slits on both sides holding the working and counter electrodes in a parallel plate configuration with Li counter and reference electrodes.

The electrolyte solution was $1 \mathrm{M} \mathrm{LiAsF}_{6}$ (Lithco) in an ethylene carbonate (EC) - dimethyl carbonate (DMC) 1:3 mixture (Merck's solvents). All other details, including glove-box operation and electrochemical measurements, were reported in previous publications $[16,17]$.

In brief, PITT and EIS were applied using Schlumberger's 1286 electrochemical interface and 1255 FRA driven by the Corrware software from Scribner Assoc. (486 IBM PC). In some cases PITT and the measurements of differential capacity as a function of electrode potential (the latter response is similar to the cyclic voltammetric response obtained at different scan rates) were performed, utilizing a multi-channel Arbin system driven by a Pentium IBM PC computer.

Highly resolved PITT and GITT data were analyzed with the use of finitediffusion models [1-3]. In the vicinity of differential capacity peaks, when necessary, the incremental potential step applied for PITT was as small as $5 \mathrm{mV}$. Each step was executed after complete equilibration during the preceding step. Residual (background) charge current was less than $0.5 \mu \mathrm{A} / \mathrm{mg}$. 

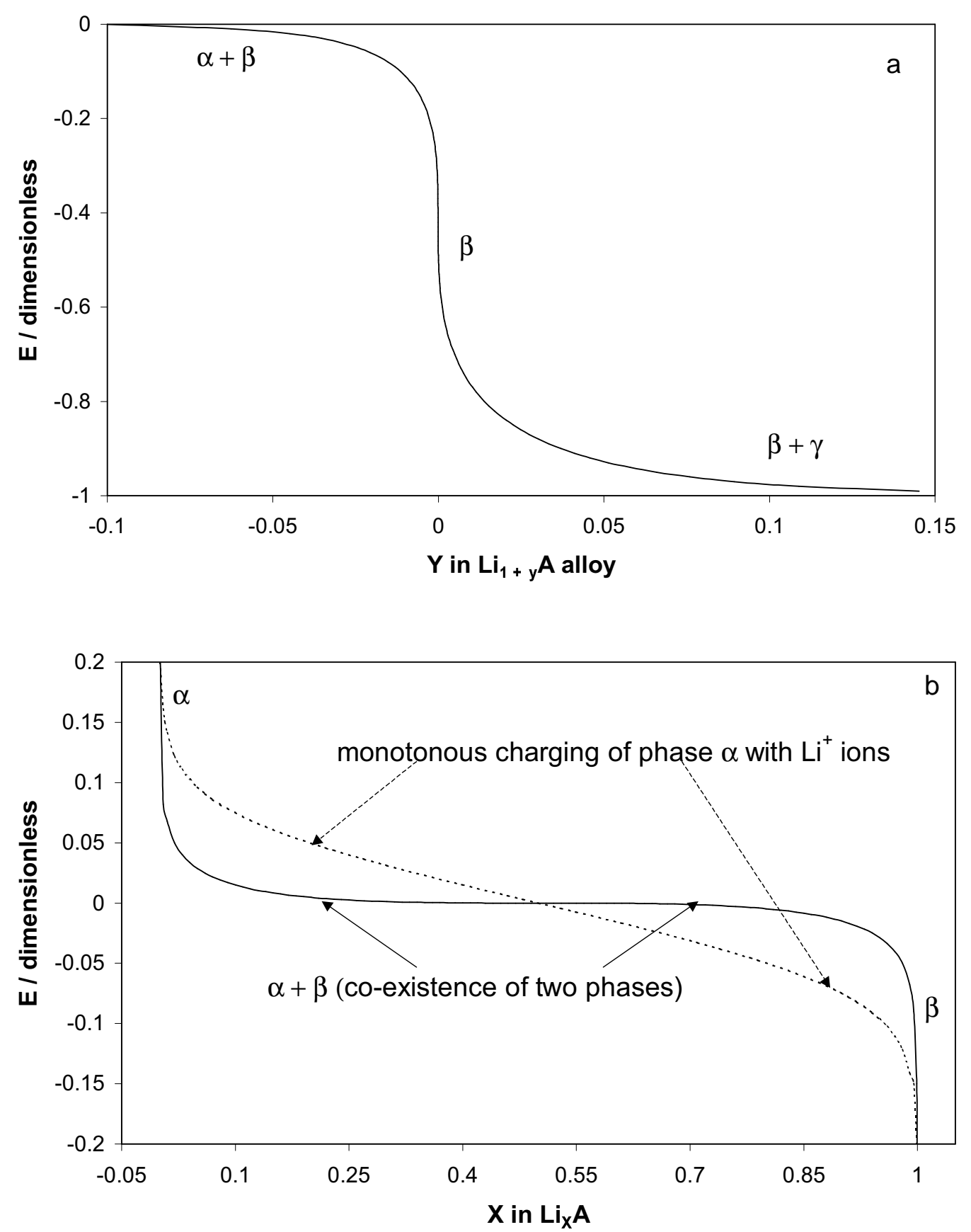

Figure 1. Schematic charging - discharging curves of $\operatorname{Li}_{1+Y} \mathrm{~A}$ alloy (a), $|Y| \ll 1$ ("dilute solution" approximation) and $\mathrm{Li}_{X} \mathrm{~A}$ intercalation compound (b), $0 \leqslant$ $X \leqslant 1$ (lattice-gas model approximation). $\alpha, \beta$ and $\gamma$ denote conventional pure phases. Solid curve in figure $1 \mathrm{~b}$ relates to a first-order phase transition between phases $\alpha$ and $\beta$, whereas broken curve corresponds to monotonous change of $X$ within $\alpha$ phase. 


\section{Results}

We consider first the case of Li-alloys, such as $\mathrm{Li}_{X} \mathrm{Al}$ and $\mathrm{Li}_{1+Y} \mathrm{Sb}$ [1,2]. Figure 1a shows schematically the equilibrium charging (discharging) curve of a Li-alloy of the conventional stoichiometry $\operatorname{Li}_{1+Y} \mathrm{~A}$. Here $X$ denotes the change in the Li-content in the bulk of the alloy, related to the drastic change in the equilibrium potential. The most interesting and well-documented kinetic feature, namely, the dramatic enhancement of the Li-ion mobility, is always observed in the very close proximity to the stoichiometric composition, e.g. $\mathrm{Li}_{1+Y} \mathrm{Sb}$ [1] where $Y$ is of the order of a few thousandths. At higher deviations from the stoichiometric composition (LiSb), the both portions of the curve relate to regions of phase co-existence, $\alpha+\beta$ or $\beta+\gamma$, at the negative or positive values of $Y$ in the $\operatorname{Li}_{1+Y} \mathrm{~A}$ (see figure 1a). Note that these two regions are usually ignored in kinetic analysis [1].

In contrast, in the case of a typical Li-intercalation compound $\operatorname{Li}_{X} \mathrm{~A}, X$ changes between 0 and 1 , and this change relates to a much more extended range of potentials compared to the potential range of interest for the Li-alloys. The solid line curve in figure $1 \mathrm{~b}$ shows schematically an equilibrium charging (discharging) curve for such an intercalation electrode with practically horizontal plateaus corresponding to the $\alpha+\beta$ phase co-existence at the intermediate intercalation levels and rather steep portions of the curve at both limits $X \rightarrow 0$ and $X \rightarrow 1$ for pure phases $\alpha$ and $\beta$, respectively. Thus the horizontal plateaus correspond to a first-order phase transition in the host bulk. The other (dashed) curve in the same figure arises in the case of the monotonous charging of phase $\alpha$. Then, the typical $X$-ray diffraction (XRD) patterns show single peaks which are shifted gradually to lower $2 \theta$ values as Liinsertion proceeds. As is presented below, our data correspond to these two different classes of $\mathrm{Li}$ insertion, namely a first-order phase transition and a smooth variation, $\mathrm{Li}_{X} \mathrm{NiO}_{2}$ and $\mathrm{Li}_{X} \mathrm{Co}_{0.2} \mathrm{Ni}_{0.8} \mathrm{O}_{2}$, respectively, being their typical representatives.

\subsection{Theoretical model of coupled electron-ion transport}

The lithium ion systems are frequently treated, mostly without any argumentation, as pure ion conductors. However, the change of the doping level of the bulk material with respect to lithium cations always requires a corresponding transfer of the electronic charge, to retain the local electroneutrality condition. It means that all these systems represent in reality mixed (electron-ion) conductors.

It is well known that the transport phenomena in such systems include a coupling between the redistributions of both mobile species, at least via the above condition. Besides, there are generally short-range interactions between these species. Depending on their character one can distinguish two limiting cases:

(1) excessive (i.e. mobile) electrons are mostly localized at the immobile centers in the host matrix so that their displacement is realized via the hopping mechanism between these centers;

(2) these electrons are mostly localized at lithium ions so that the system can be modeled as mobile lithium atoms inside the host matrix. 
Both classes of systems can be described by similar sets of equilibrium and transport equations but with different assumptions on the values of the parameters in them. In this paper the consideration is limited to type (1).

The general transport equations for mixed conductors inside a solid matrix may be based on the irreversible thermodynamics or on "multi-component diffusion" approach [18]. If charging (discharging) of $\mathrm{Li}_{1+Y} \mathrm{~A}$ alloy or $\mathrm{Li}_{X} \mathrm{~A}$ intercalation compound is performed using small-amplitude perturbations from their equilibrium states, then a linear response theory can be applied. Then, one can linearize the equations with respect to the perturbations of all variables. In particular, there exist linear relations between the gradient of the perturbation of the electrochemical potential $\mu_{\alpha}$ of a species $\alpha$ (electrons or ions) and the instantaneous flux densities of all species $J_{\beta}$ :

$$
c_{\alpha}^{\circ} \nabla \mu_{\alpha}=\sum_{\beta} K_{\alpha \beta}\left(J_{\beta} / c_{\beta}^{\circ}-J_{\alpha} / c_{\alpha}^{\circ}\right)
$$

Here, index $\alpha$ means $e$ or $i$ (electrons or Li cations), $\beta$ runs all interacting components, electrons, ions and the host matrix $(m), K_{\alpha \beta}$ are the "friction coefficients" characterizing the interaction between the corresponding fluxes which are generally some functions of unperturbed concentrations $c_{\alpha}^{\circ}$, this matrix being symmetrical, $K_{\alpha \beta}=K_{\beta \alpha}$.

In the coordinates with respect to the matrix its velocity is zero and the equations can be rewritten as [19]:

$$
\begin{aligned}
& \nabla E_{e} \equiv \nabla\left(\mu_{e} / z_{e} e\right)=-k_{e} i_{e}+k i_{i}, \\
& \nabla E_{i} \equiv \nabla\left(\mu_{i} / z_{i} e\right)=-k_{i} i_{i}+k i_{e},
\end{aligned}
$$

where the fluxes are replaced by the partial current densities, $i_{\alpha} \equiv z_{\alpha} e J_{\alpha}$ ( $e$ is the proton charge, the charge valency of ion, $z_{i}$, is chosen below as +1 while $z_{e}=$ $-1)$. The electrochemical potentials are proportional to the corresponding electrode potentials, $E_{e}$ or $E_{i}$, i.e. the local values of the potential measured with an electrode reversible with respect to the corresponding species, $e$ or $i$.

Coefficients $k_{e}, k_{i}$ and $k$ represent combinations of three coefficients $K_{\alpha \beta}$ in equations (1). In particular, $k$ is proportional to the non-diagonal term, $K_{e i}$, in equations (1). Since it is assumed in this paper that the electrons are mostly localized at the host lattice, this term will be neglected, see for discussion in [6]. Within this approximation the diagonal coefficients in equations $(2 \mathrm{a}),(2 \mathrm{~b})$ are directly related to the partial electron and ion conductivities, $k_{\alpha}=1 / \kappa_{\alpha}$ :

$$
i_{e}=-\kappa_{e} \nabla E_{e}, \quad i_{i}=-\kappa_{i} \nabla E_{i} .
$$

One can introduce two other parameters instead of these characteristics, total conductivity $\kappa$ and the transport numbers of electrons and ions $t_{e}, t_{i}$ :

$$
\kappa=\kappa_{e}+\kappa_{i}, \quad t_{e}=\kappa_{e} / \kappa, \quad t_{i}=\kappa_{i} / \kappa, \quad t_{e}+t_{i}=1 .
$$

Another important characteristic of the system is its redox capacitance, $C_{\text {int }}$. It is defined as the ratio of the specific charge to be supplied to the film across its 
interfaces in quasi-equilibrium conditions to the change of the electrode potential, $\mathrm{d} E$

$$
C_{\text {int }}=-\mathrm{d} \rho_{i} / \mathrm{d} E \equiv-V^{-1} \mathrm{~d} Q_{i} / \mathrm{d} E
$$

The electron and ion charges inside the film, $Q_{e}$ and $Q_{i}$, are proportional to their charge densities, $\rho_{e, i}$, or their occupation numbers per unit site in the matrix, $X_{e}$ and $X$ :

$$
Q_{e} \equiv-V \rho_{e} \equiv-Q_{m} X_{e}, \quad Q_{i} \equiv V \rho_{i} \equiv Q_{m} X, \quad Q_{m}=N_{\mathrm{A}} e V / V_{m}
$$

Here, $N_{\mathrm{A}}$ is the Avogadro number, $V$ the volume of the system, $V_{m}$ the molar volume, $Q_{m}$ the maximum (limiting) intercalation charge (if $X$ varies between 0 and 1 ). The electroneutrality condition for the bulk film may be written in two forms:

$$
Q_{e}+Q_{i}+Q_{s}=0, \quad \text { i.e. } \quad X_{e}=X+z_{s},
$$

where $Q_{s}$ is the "fixed" charge of the host matrix, $z_{s}=Q_{s} / N_{\mathrm{A}} e$ is the corresponding charge valency of a site. The charge valencies of electrons and mobile ions (lithium cations) is taken as -1 and +1 , correspondingly.

The electrode potential in the equilibrium is directly related to the difference of electron and ion electrode potentials inside the film:

$$
E=E_{e i}+\text { const }, \quad E_{e i} \equiv E_{e}-E_{i} \equiv-\left(\mu_{e}+\mu_{i}\right) / e+\text { const. }
$$

Thus, to determine the redox capacitance it is sufficient to modelize the dependences of the chemical potentials of electrons and ions in equation (8) (since the electric terms are cancelled in $E_{e i}$ ) on the charging degree, $X$ :

$$
C_{\mathrm{int}}=-Q_{m} V^{-1} \mathrm{~d} X / \mathrm{d} E_{e i} \equiv-e Q_{m} V^{-1} \mathrm{~d} X / \mathrm{d}\left(\mu_{e}+\mu_{i}\right) .
$$

This formula shows that the intercalation capacitance is determined by the internal properties of this phase, i.e. it is independent of its interfacial structure or other phases.

The constant terms in equation (8) depend on the type of the reference electrode. If it is lithium metal, then

$$
E^{\mathrm{M} / \mathrm{Li}}=e^{-1}\left(\mu_{\mathrm{Li}}-\mu_{e}-\mu_{i}\right)
$$

where $\mu_{\mathrm{Li}}$ is the chemical potential of lithium atom in the metal phase. On the other hand, if the reference electrode is reversible with respect to the anion, $\mathrm{A}^{-}$, of the binary electrolyte in the solution, LiA, equation (8) takes the form:

$$
E^{\mathrm{M} / \mathrm{A}}=e^{-1}\left(\mu_{\mathrm{LiA}}-\mu_{\mathrm{A}}-\mu_{\mathrm{e}}-\mu_{\mathrm{i}}\right) .
$$

Now, $\mu_{\mathrm{LiA}}$ is the chemical potential of the LiA salt in the solution $\left(\mu_{\mathrm{LiA}} \equiv \mu_{\mathrm{Li}}^{+}+\mu_{\mathrm{A}}^{-}\right)$, $\mu_{\mathrm{A}}$ the chemical potential of the neutral $X$ species in the reference electrode, e.g. $\mu_{\mathrm{Cl}} \equiv \mu_{\mathrm{AgCl}}-\mu_{\mathrm{Ag}}$ for the $\mathrm{Ag} / \mathrm{AgCl}$ electrode. 
In the latter case one can vary the electrolyte concentration, i.e. the chemical potential of the salt LiA, while keeping the electrode potential versus this anion reference electrode, $E^{\mathrm{M} / \mathrm{A}}$, fixed. The dependence of the lithium intercalation charge as a function of this chemical potential, $Q_{i}\left(\mu_{\mathrm{LiA}}\right)$, for $E^{\mathrm{M} / \mathrm{A}}=$ const may be called intercalation isotherm.

The combination of equations ( $8 b$ ) and (9) gives a simple relation between the intercalation capacitance and the isotherm:

$$
\begin{aligned}
C_{\text {int }} & \equiv-Q_{m} V^{-1} \mathrm{~d} X / \mathrm{d} E=-e Q_{m} V^{-1} \mathrm{~d} X / \mathrm{d}\left(\mu_{e}+\mu_{i}\right) \\
& \equiv e V^{-1} \mathrm{~d} Q_{i} / \mathrm{d} \mu_{\mathrm{LiA}}, \\
E^{\mathrm{M} / \mathrm{A}} & =\text { const. }
\end{aligned}
$$

This formula also demonstrates a proportionality of the derivatives of the dependences, $X(E), X\left(\mu_{e}+\mu_{i}\right)$ and $Q_{i}\left(\mu_{\mathrm{LiA}}\right)$. In this meaning the former may also be termed as the "intercalation isotherm".

Let us introduce now another transport characteristics, $D_{e i}$, which is called binary or electron-ion or chemical diffusion coefficient. It may be defined from the relation between the fluxes and the concentration gradients, or between the partial currents and the gradients of the corresponding charge density, at the condition of the zero overall current, $i=i_{e}+i_{i}$ (the displacement current is neglected in this paper):

$$
i_{e}=-D_{e i} \nabla \rho_{e}, \quad i_{i}=-D_{e i} \nabla \rho_{i} \quad \text { for } \quad i=0
$$

(these two definitions are equivalent since $i_{e}+i_{i}=0$ and $\nabla \rho_{e}+\nabla \rho_{i}=0$ ).

In the absence of the non-diagonal term in equations (2) (see [19] for the general case) one can get a relation between this diffusion coefficient and the partial conductivities or transport numbers.

For it one can derive a general relation for the partial currents. A combination of equations (3), (8), (9) and (6) gives:

$$
i_{e} / t_{e}-i_{i} / t_{i}=-\kappa \nabla E_{e i}=-\kappa C_{\mathrm{int}}^{-1} \nabla \rho_{e} .
$$

Since the sum of the partial current is equal to the overall one, i, one can find both partial currents:

$$
i_{e}=t_{e} i-t_{e} t_{i} \kappa C_{\mathrm{int}}^{-1} \nabla \rho_{e}, \quad i_{i}=t_{i} i-t_{e} t_{i} \kappa C_{\mathrm{int}}^{-1} \nabla \rho_{i} .
$$

The comparison with equation (10) for $i=0$ gives relations between $D_{e i}$ and the total or partial conductivities:

$$
D_{e i}=t_{e} t_{i} \kappa C_{\mathrm{int}}^{-1} \equiv t_{e} \kappa_{i} C_{\mathrm{int}}^{-1} \equiv t_{i} \kappa_{e} C_{\mathrm{int}}^{-1} .
$$

This relation in one of these forms has appeared in many publications, see e.g. $[1,4]$. The above derivation emphasizes that this formula is quite symmetrical with respect to electrons and ions, in particular the above diffusion coefficient represents a joint property of both mobile species and any of the partial conductivities may be introduced in this relation. 
A similar relation between the diffusion coefficient and the conductivity also exists for materials possessing a single type of mobile charge carriers, either electronic or ionic ones [20-22]:

$$
D=\kappa e^{-2} \mathrm{~d} \mu_{o} / \mathrm{d} c
$$

$\mu_{o}$ being the chemical potential, an analog of $\mu_{e}+\mu_{i}$ in equation (8).

Often equation (13) is written down in a different form for systems where the ion transport is of primary importance while the electron one represents an inevitable coprocess. The partial ion conductivity is transformed in the way as if the mobile ions can be considered within the framework of the ideal solution approximation where the conductivity and the diffusion coefficient are related by the Nernst-Einstein formula:

$$
\kappa_{i} \equiv c_{i} N_{\mathrm{A}} e^{2}(k T)^{-1} D_{i} .
$$

In the general case where the ion system cannot be treated as an ideal solution this equation represents a definition of the quantity which is called [1] the component diffusion coefficient. In this meaning its value can only be found experimentally from the ion conductivity measurements. In particular, it has no relation to the measurements of the diffusion rates in PITT or GITT conditions which allows one to determine the chemical (electron-ion) diffusion coefficient $D_{e i}$.

The combination of equations (13) and (14) gives for the ratio of these two diffusion coefficients:

$$
D_{e i}=W_{i} D_{i}, \quad W_{i}=t_{e} c_{i} N_{\mathrm{A}} e^{2}(k T)^{-1} C_{\text {int }}^{-1} .
$$

The multiplier, $W_{i}$, in this formula is called Wagner or enhancement factor [1]. Equation (15) relates it to the electron transport number and the redox capacitance.

For the intercalation phase of the $\operatorname{Li}_{1+Y}$ A alloy, $c_{i}=X Q_{m}\left(V N_{\mathrm{A}} e\right)^{-1}, X \equiv 1+Y$, and this formula for $W_{i}$ takes the form after the use of equation (9):

$$
W_{i}=-\left(t_{e} e / k T\right) X \mathrm{~d} E / \mathrm{d} X \equiv(k T)^{-1} t_{e} X \mathrm{~d}\left(\mu_{e}+\mu_{i}\right) / \mathrm{d} X .
$$

From figure 1a we can see that in the vicinity of the composition related to the stoichiometric alloy LiA, the derivative, $\mathrm{d} E / \mathrm{d} X$, is rather high, thus the chemical diffusion coefficient $D_{e i}$ may be several orders of magnitude greater than the ion ("component") diffusion coefficient, $D_{i}$.

The use of the identity,

$$
\mathrm{d}\left(\mu_{e}+\mu_{i}\right) \equiv k T \mathrm{~d} \ln \left(a_{e} a_{i}\right) \equiv k T \mathrm{~d} \ln a_{\text {atom }}
$$

$\left(a_{e}, a_{i}, a_{\text {atom }}\right.$ are the activities of electrons, mobile ions and corresponding atoms inside the intercalation phase) allows one to arrive to an equivalent formula for $W_{i}$ [1]:

$$
W_{i}=t_{e} \mathrm{~d} \ln a_{\text {atom }} / \mathrm{d} \ln c_{i} .
$$

One should keep in mind that this introduction of the atomic activity has no relation to the real existence of lithium atoms in the system. Moreover, the neglect of the non-diagonal terms in equations (1) which represents the basis of this formula can 
be justified just for the systems where the association of mobile electrons and ions into atoms is practically absent.

In view of the symmetry of equation (13) quite a similar "enhancement factor", $W_{e}$, can also be introduced for electrons satisfying to analogous equations.

We'll see below that the ion enhancement factor represents a convenient quantity for the ion transport in continuous media. On the other hand, for lithium diffusion inside a solid lattice where the saturation effects play a crucial role one can introduce a more suitable characteristic of the system based on a different definition of the "ion diffusion coefficient", $D_{i}^{\circ}$, instead of $D_{i}$ in equation (14):

$$
\kappa_{i} \equiv c_{i}\left(1-c_{i} / c_{m}\right) N_{\mathrm{A}} e^{2}(k T)^{-1} D_{i}^{\circ} \equiv X(1-X) V_{m}^{-1} N_{\mathrm{A}} e^{2}(k T)^{-1} D_{i}^{\circ}
$$

where $c_{m} \equiv V_{m}^{-1}$ the maximum concentration of intercalated ions. It leads to a "modified enhancement factor", $W_{i}^{\circ}$, defined by equation (15a):

$$
D_{e i}=W_{i}^{\circ} D_{i}^{\circ}, \quad W_{i}^{\circ}=t_{e} X(1-X) V_{m}^{-1} N_{\mathrm{A}} e^{2}(k T)^{-1} C_{\text {int }}^{-1} .
$$

Then, equations (5) and (9) give for this factor:

$$
W_{i}^{\circ}=-\left(t_{e} e / k T\right) X(1-X) \mathrm{d} E / \mathrm{d} X \equiv(k T)^{-1} t_{e} X(1-X) \mathrm{d}\left(\mu_{e}+\mu_{i}\right) / \mathrm{d} X .
$$

It is shown below that these new quantities, $D_{i}^{\circ}$ and $W_{i}^{\circ}$, represent more informative characteristics of the intercalation system.

Let us apply these relations to particular models of ionic systems.

\section{2. "Dilute solution" approximation} $[1-3]$.

The basic results for this model have been derived by Weppner and Huggins

"Dilute solution" approximation is based on the expression for the electrochemical potential which is used for both electron and ion species in equation (8):

$$
\mu_{\alpha}=\mu_{\alpha}^{\circ}+k T \ln c_{\alpha}+z_{\alpha} e \phi
$$

where $\mu_{\alpha}^{\circ}$ stands for the chemical potential in the standard state, $\phi$ is the electrostatic potential, $k$ and $T$ are the Boltzmann constant and the absolute temperature. Besides, the partial ion conductivity $\kappa_{i}$ is proportional to the ion electric mobility $u_{i}$ or the ion diffusion coefficient, $D_{i}$ which both are concentration independent:

$$
\kappa_{i}=N_{\mathrm{A}} e c_{i} u_{i}=N_{\mathrm{A}} e^{2}(k T)^{-1} c_{i} D_{i} .
$$

The latter formula is identical to equation (14), i.e. in this case the quantity introduced by equation (14) corresponds to the real ion diffusion coefficient. Then, one can use general equations $(15)$ and $\left(15^{\prime}\right)$ for the enhancement factor, $W_{i}$. The combination of equations (15) and (17) (for the electrons and ions) leads to the expression:

$$
W_{i}=t_{e}\left(1+c_{i} / c_{e}\right)
$$


Note that equation (19) corresponds to the case of single-charged cations and electrons. In general case, where the charge number of the electronic species is equal to $z_{e}$ and the corresponding charge number of ions is $z_{i}$, then this formula takes the form:

$$
W_{i}=t_{e}\left(1+z_{i}^{2} c_{i} / z_{e}^{2} c_{e}\right) .
$$

Equation (19) is of crucial importance for understanding the physical meaning of $W_{i}$ when turning from $\mathrm{Li}_{1+Y} \mathrm{~A}$ alloy to $\mathrm{Li}_{X} \mathrm{~A}$ intercalation compound.

The enhancement factor $W_{i}$ is expected to be close to 1 in the two following particular cases: (i) when the mobility of electrons is much higher than that of ions (so that $t_{e} \cong 1$ ), and the host contains an excess of electrons $\left(c_{e} \gg c_{i}\right)$, (ii) when one can use "ideal solution" formula (18) for electrons, too, besides $D_{e}=D_{i}$ (then, $\left.t_{e}=0.5\right)$ and $c_{e}=c_{i}$. On the contrary, if the electron and ion concentrations are equal, $c_{e}=c_{i}$ (it means the absence of the "fixed charge", $Q_{s}$, in equation (7)) but the conductivity of electrons is much higher than that of ions (i.e. $D_{e} \gg D_{i}$ ), then equation (19) results in $t_{e}=1$ and $W_{i}=2$.

Finally, for certain semiconductors, the concentration of electrons can be lower than that of ions, $c_{e} \ll c_{i}$, whereas their conductivity is, in contrast, much higher than that of ions, such that $t_{e} \cong 1$. In this case, $W_{i}$ may easily increase up to $10^{3}-10^{4}$ which is typical for the various $\operatorname{Li}_{1+Y} \mathrm{~A}$ alloys $[1,2]$.

Thus, equation (19) is useful for the comparison of electrochemical behaviour of $\mathrm{Li}_{1+Y} \mathrm{~A}$ alloy and $\mathrm{Li}_{X} \mathrm{~A}$ intercalation compound (such as those shown in figures 1a and $1 \mathrm{~b})$. However, we should remember that the expression for the "enhancement factor" $W_{i}$ in equation (19) was derived in the "dilute solution" approximation. In the following section we will see how the "saturation effect" in the course of Li-ion intercalation into inorganic hosts influences these predictions for the "enhancement factor" $W_{i}$.

\section{3. "Saturation effect" and the "enhancement factor"}

Consider first the case of monotonous charging occurring in an inorganic host within the whole range of the intercalation level, from 0 to 1 (see broken line in figure 1b). Two particular cases are dilute solutions (at small values of $X$ ) and "saturation limit" (at $X$ close to 1), respectively. The "saturation limit" originates from a fixed number of sites in the host, accessible for Li-ion intercalation. Similar to thermodynamic description of holes in conventional inorganic semiconductors, the electrochemical potential of Li-ions can be represented as a simple logarithmic function of the entropy factor $X /(1-X)$, i.e. the function of the ratio of the number of occupied sites to the number of unoccupied ones:

$$
\mu_{i}=\mu_{i}^{\circ}+k T \ln \{X /(1-X)\}+e \phi .
$$

To simplify the further equations, the "ideal solution" approximation, equation (17), will be retained for the chemical potential of electrons. Then, equations (5), (6) and (8) give the expression for the redox capacitance:

$$
C_{\text {int }}=N_{\mathrm{A}} e^{2}(k T)^{-1}\left\{c_{e}^{-1}+c_{i}^{-1}(1-X)^{-1}\right\}^{-1} .
$$


Then, formula (15) leads to the expression for the "enhancement factor":

$$
W_{i}=t_{e}\left\{(1-X)^{-1}+c_{i} / c_{e}\right\} \text {. }
$$

Assuming that $t_{e}=1$ and a high concentration of electrons, $c_{e} \gg c_{i}$, the "enhancement factor" is reduced to $W_{i}=(1-X)^{-1}$. This expression is definitely valid in "dilute solution" approximation: at small levels of intercalation $W_{i}=1$ and the chemical diffusion coefficient is close to the ion one, $D_{i}$. However, no meaningful explanation can be offered for the infinite increase of $W_{i}$ as $X$ approaches unity. The reason of this failure is related to the lattice-gas model for the distribution of intercalation sites in which the electrical mobility of Li-ions $u_{i}$ should not be taken as a constant at high values of $X$, in contrast to the case of small $X$. Thus, a proper model for the dependence of $u_{i}$ on $X$ is required to adequately describe kinetic properties of intercalation compounds.

\subsection{The "enhancement factor" and intercalation isotherms}

One can derive another expression for the chemical (electron-ion) diffusion coefficient $\mathrm{D}_{e i}$ with the use of equations (13), (5), (6) and (8):

$$
D_{e i}=t_{e} e^{-1} u_{i} X \mathrm{~d} \mu_{e i} / \mathrm{d} X, \quad \mu_{e i}=\mu_{e}+\mu_{i} .
$$

This formula is still valid for a general case, except for the assumed proportionality of $c_{i}$ and $X$.

One needs now to specify the dependence of the ion mobility, $u_{i}$, and the total (electron-ion) chemical potential from the charging degree.

For the first problem one can use the argumentation derived for the systems with a pure ion conductivity [22]. If the ion subsystem is close to the saturation level one can consider this saturation limit (all possible sites are occupied by lithium cations) as the background point, to which a dilute lattice gas of vacancies in this cation lattice is added. Then, the ion conductivity must be proportional to the concentration of these vacancies, $\kappa_{i} \sim\left(c_{m}-c_{i}\right)$, where the maximum concentration, $c_{m}$, is defined in equation (14a). On the other hand, for low charging levels, the conductivity is proportional to the ion concentration, $c_{i}$. Thus, the general expression for the conductivity of intercalated ions must have the form [22]:

$$
\kappa_{i} \equiv c_{i}\left(1-c_{i} / c_{m}\right) N_{\mathrm{A}} e^{2}(k T)^{-1} D_{i}\left(c_{i}\right),
$$

where the constant multiplier, $N_{\mathrm{A}} e^{2}(k T)^{-1}$, is chosen to ensure that the unknown function, $D_{i}\left(c_{i}\right)$, approaches to the "component" (ion) diffusion coefficients of the ions or of the vacancies for low or high values of the charging level, $X \ll 1$ or $1-X \ll 1$.

If the transition of an ion between the neighbouring sites in the lattice is independent of the presence of the other ions (except for their blocking effects of the sites), then this function, $D_{i}\left(c_{i}\right)$, represent a constant, $D_{i}^{\circ}$, and the general expression $(24)$ is reduced to formula (14a). 
This expression (14a) for the ion conductivity explains the reason of an unexpected behaviour of the conventional "enhancement factor", $W_{i}$, in equation (22). Comparison of equations (14) and (14a) shows that the formally introduced "component diffusion coefficient", $D_{i}$, represents a function of the ion concentration:

$$
D_{i}=D_{i}^{\circ}(1-X)
$$

i.e. this quantity tends to zero near the saturation limit. As a result, the conventional enhancement factor defined by equation (15) increases as $(1-X)^{-1}$, to compensate this behaviour.

On the contrary, this singularity is removed in the definition of the new "enhancement factor", $W_{i}^{\circ}$, defined in equation (15a) which rests finite for both limits of low and high charging levels.

As an illustration, one can insert expression (21) for the redox capacitance into equation (15a) to obtain:

$$
W_{i}^{\circ}=t_{e}\left\{1+X(1-X) c_{m} / c_{e}\right\}
$$

Its value is close to 1 for the whole range of the intercalation levels if both electron concentration and its conductivity are much higher than those for ions.

Another important formula relates the electron-ion (chemical) diffusion coefficient and the "adsorption isotherm". Combination of equations (24) and (23) results in the relation:

$$
D_{e i}=t_{e} X(1-X) D_{i}^{\circ}(k T)^{-1} \mathrm{~d} \mu_{e i} / \mathrm{d} X, \quad \mu_{e i}=\mu_{e}+\mu_{i} .
$$

Its approximate form for the particular case corresponding to the dominance of the electronic subsystem (both concentration and conductivity):

$$
D_{e i}=X(1-X) D_{i}^{\circ}(k T)^{-1} \mathrm{~d} \mu_{i} / \mathrm{d} X
$$

was earlier used by McKinnon to emphasize the relation between $D_{e i}$ and intercalation isotherms [6].

A simplest Frumkin-type intercalation isotherm with an interaction constant $g$ (which is independent of $X$ ) has the following form [23,24]:

$$
(e / k T) E=g X+\ln (X /(1-X)) .
$$

The expression for the chemical potential of Li-ions corresponding to this isotherm takes into account both the "saturation effect" and interaction between the intercalation sites:

$$
\mu_{i}=\mu_{i}^{o}+k T \ln (X /(1-X))+k T g X .
$$

Differentiating $\mu_{i}$ with respect to $X$ in equation (29) and combining further the result with equation (27) allows for derivation of the final expression for $D_{e i}$ valid for the whole range of intercalation levels from 0 to $1[4,24]$ :

$$
D_{e i}=D_{i}^{\circ}[1+g X(1-X)]
$$


Equation (30) defines the new "enhancement factor" $W_{i}^{\circ}=D_{e i} / D_{i}^{\circ}$ which is related to the intercalation isotherm, equation (28):

$$
W_{i}^{\circ}=[1+g X(1-X)] .
$$

Comparing two "enhancement factors", namely the classical one, $W_{i}$ (equation (19) and that related to the intercalation isotherm, $W_{i}^{\circ}$ (equation (26)), we should note that they reflect quite different characteristics of mixed-conduction electrodes. Elimination of the potential gradient $\partial \phi / \partial y$ in equation (3) through the bulk electroneutrality condition (equation (7)) resulted in the factor $W_{i}$, which reflected the dependence of the ionic flux density $j_{i}$ on the ratios of electronic and ionic mobilities and concentrations in the "dilute solution" approximation. In contrast, equation (31) describes mainly the dependence of the new factor $W_{i}^{\circ}$ on the interaction parameter $g$ in the whole range of intercalation levels $X$. It is seen that independent of $g, W_{i}^{\circ}=1$ both at very small $X$ and close to saturation, $X \rightarrow 1$. For these two regions $W_{i}=W_{i}^{\circ}=1$ since as we will show in the next section a simple Frumkin intercalation isotherm with one single potential drop across the host/solution interface formally corresponds to $c_{i} / c_{e} \ll 1$ and $t_{e}=1$, thus according to equation $\left(19^{\prime}\right)$ $W_{i}=1$. The physical meaning of $W_{i}^{\circ}$ is quite different from that of $W_{i}$. Since according to equation (31) $W_{i}^{\circ}=1$ at $g=0$ within the whole range of $X$, the deviation of $W_{i}^{\circ}$ from unity reflects the deviation of the related chemical diffusion coefficient from the true constant component diffusion coefficient caused by interactions between the intercalation sites. As was discussed elsewhere, for attractive interactions $(g<0)$, $W_{i}^{\circ}<1$ whereas for repulsive interactions $(g>0) W_{i}^{\circ}>1$. In the case when the attractive interaction reaches a critical value $g_{\text {crit }}=-4, W_{i}^{\circ}=0$. Within two-phase co-existence region (as related to solid line in figure $1 \mathrm{~b}$ ), $W_{i}^{\circ}$ takes formally negative values.

It follows from the second expression of equation (13) for $t_{e} \cong 1$ that the ionic conductivity of the intercalation electrodes can be written in the following way (similar to the parameterization of the electronic conductivity of electroactive redoxpolymers, see [4]):

$$
\kappa_{i}=C_{\mathrm{int}} D_{e i}=\left(Q_{m} / V\right) D_{e i}(\mathrm{~d} X / \mathrm{d} E),
$$

where $V$ is the volume of the intercalation electrode under study, $Q_{m}$ is the limiting (maximum) intercalation charge and $C_{\text {int }}$ is the differential intercalation capacity an important equilibrium characteristic of the intercalation electrode. The quantity $C_{\text {int }}$ can be easily derived by taking the reciprocal of the derivative of the electrode potential $E$ with respect to $X$ (the Frumkin isotherm, equation (28)):

$$
C_{\text {int }}=\left(e Q_{m} / k T\right)\left[g+X^{-1}+(1-X)^{-1}\right]^{-1} .
$$

Substitution of $C_{\text {int }}$ from equation (33) to equation (33) results finally in the following expression for $\kappa_{i}$ :

$$
\kappa_{i}=\left(e Q_{m} D_{i}^{\circ} / k T V\right) X(1-X) .
$$

Equation (34) coincides with equation (14a) if to take into account the dependence of $D_{i}^{\circ}$ on $c_{i}$. Surprisingly, it appears that $\kappa_{i}$ is not an explicit function of $g$. This 
is due to the fact that $C_{\text {int }}$ (equation (33)) is inversely proportional to $W_{i}^{\circ}$ whereas $D_{e i}$ depends linearly on $W_{i}^{\circ}$ (equations (30) and (31), thus the dependence of $\kappa_{i}$ on $g$ is cancelled. However, $g$ will implicitly affect the dependence of $\kappa_{i}$ on $E$ since $X=X(g, E)$, see equation (28). Alternatively, $\kappa_{i}=\kappa_{i}(X)$ relationships can be converted to the related $\kappa_{i}=\kappa_{i}(E)$ relationships by changing $X$ for $E$ using for this numerical integration of the reciprocal of $C_{\text {int }}$ :

$$
E-E_{o}=\left(e Q_{m} / k T\right) \int C_{\text {int }}^{-1} \mathrm{~d} X .
$$

Equations (33)-(35) are used in this work for theoretical simulation of $\kappa_{i}$ vs. $E$ curves. On the other hand, equation (32) shows that $\kappa_{i}$ can be easily determined experimentally as a function of $E$ due to the fact that both these quantities $D_{e i}$ and $C_{\text {int }}$ are calculated using any incremental titration technique such as potentiostatic or galvanostatic intermittent titration (PITT and GITT, respectively) or electrochemical impedance spectroscopy (EIS). We have already discussed in full detail the particular features of the related experimental procedures appearing during determination of $D_{e i}$ and $C_{\text {int }}$ for electrodes with highly attractive interactions between the intercalation sites [24]. Here we only refer to the final form of the equation, which can be used for the experimental determination of $\kappa_{i}$ by PITT:

$$
\kappa_{i}=\left(\pi l^{2} / V Q_{m} \Delta X\right)\left[\left(I t^{1 / 2}\right)^{2} / \Delta E\right]
$$

where $l$ is the characteristic diffusion length in the finite-space diffusion model. For thin-film electrodes $l$ is equal to the film's thickness whereas for porous powdery electrodes $l$ rather relates to half of the average particle size. $\Delta E$ is an applied small potential increment during which the measurement of the instantaneous current is carried out. Next potential increment is applied when the current drops to a very small value (equilibrium state). $\Delta X$ is the change of the intercalation level related to the applied potential increment $\Delta E$.

\subsection{Relation of the classical "enhancement factor" $W_{i}$ to particular forms of intercalation isotherms}

The use of the classical "enhancement factor" $W_{i}$ in describing solid-state diffusion is relevant only in "dilute solution" approximation and, in fact, $W_{i}$ cannot reflect interactions between the intercalation sites. However, this factor may be related to the particular form of the intercalation isotherm relevant to a specific system. Such a relation is based on the fact that both the isotherm and $W_{i}$ are functions of the ratio of the bulk concentrations of the electronic and ionic species in the electroactive material and the stoichiometry of the related association process, which forms the neutral species as the final result of the insertion process. A detailed analysis of the shape of isotherms for different stoichiometries of association reactions in the bulk of electroactive polymer films has been reported previously [25]. Here we will refer to several simple examples, which can also be relevant to intercalation reactions in inorganic hosts. In accordance with equation (19) when the host material is highly electronically conducting $\left(c_{e} \gg c_{i}\right.$, and $u_{e}>u_{i}$ and hence $\left.W_{i}=1\right)$, a complete 
electronic equilibrium takes place across the current collector/host bulk interface. Therefore, this potential drop will not change with applied potential (the changes in electron concentration with potential are much smaller than the bulk electron concentration itself). This system is characterized by a single potential drop across the host/solution interface due to the dependence of the chemical potential of Li-ions on $X$, equation (29). In this case a simple Frumkin-type isotherm (equation (28)) rigorously describes the $E$ vs. $X$ relationship; the related differential intercalation capacity is expressed as a function of $X$ by equation (33).

Let us consider another case, which frequently takes place for the hosts possessing semiconductor properties. If $u_{e}>u_{i}$, as in the former case, but $c_{e}=c_{i}$, then $t_{e}=1$ and $W_{i}=2$ (see equation (19)). Taking into account both the Li-ion and electron species and assuming 1:1 ratio of their concentrations during the intercalation we come to conclusion that the both interfaces, i.e. the current collector/host and host/solution interface, should be polarized, and the changes in the interfacial potentials across these interfaces should be equal [25]. In this case the simple Frumkin isotherm is no more valid, and we have to use a more general equation instead of equation $(28)$ [4,23]:

$$
(e / k T) E=g X+\ln (X /(1-X))-z_{i}^{-1} \ln \left[\left(1-X-z_{s}\right) / z_{i}\right] .
$$

The differential intercalation capacity corresponding to this isotherm can be written with a certain modification of equation $(33)[4,23]$ :

$$
C_{\mathrm{int}}=\left(e Q_{m} / k T\right)\left\{g+X^{-1}+\left[(1-X)^{-1}\right]\left[z_{i} /\left(1-X-z_{s}\right)\right]\right\}^{-1} .
$$

Here $z_{e}$ and $z_{i}$ are the charge numbers of the electronic and ionic species whereas $z_{s}$ is their ratio: $z_{s}=-z_{i} z_{e}$. Equation (37) describes explicitly different forms of intercalation isotherms as a function of $z_{i}$, i.e. the function of the bulk material stoichiometry. In particular, the case $W_{i}=2$ may relate to $z_{e}=-1, z_{i}=1$ and $z_{s}=1$. As a result, equations (37) and (38) are simplified to the following form:

$$
(e / k T) E=g X+\ln \left[X^{2} /(1-X)\right]
$$

and

$$
C_{\text {int }}=\left(e Q_{m} / k T\right)\left[g+X^{-1}+X^{-1}(1-X)^{-1}\right]^{-1} .
$$

Earlier equations similar to (39) and (40) were successfully applied for fitting to the experimental $C_{\text {int }}$ vs. $E$ curves obtained with thin films of conventional redox [4] and electronically conducting polymers [25]. One important particular case relates to the bipolaronic (electronic) species in the bulk of conducting polymers: $z_{e}=2, z_{s}=0.5$ (for $z_{i}=-1$ ). The electroneutrality condition in this case requires substitution of $z_{i}$ for $z_{s}$ in equation $\left(19^{\prime}\right)$. Since $c_{i} / c_{e}$ is obviously 2 in this case, then $W_{i}=1.5$. In accordance with this value it was previously established that the isotherm for the bipolaronic species coincides with equation (39) with substitution of the second term in its right-hand side $\ln \left[\left(X^{2} /(1-X)\right]\right.$ for $\ln \left[\left(X^{1.5} /(1-X)\right]\right.$.

Recently, we tried to apply equation (39) for its fitting to experimental charging curves related to a powdery spinel electrode [26]. 
A more general expression for the chemical diffusion coefficient of ions can be obtained by the modification of equation (30) [4]:

$$
D_{e i}=D_{i}^{\circ}\left\{1+\left[g-z_{i}^{-1}\left(X-z_{s}\right)^{-1}\right] X(1-X)\right\} .
$$

In the particular case of $W_{i}=2\left(z_{s}=1, z_{i}=1, t_{e}=1\right)$ equation (41) is reduced to the form:

$$
D_{e i}=D_{i}^{\circ}\left\{1+\left[g+(1-X)^{-1}\right] X(1-X)\right\} .
$$

Equations (39), (40) and (42) are used in combination with equation (32) in the following section for theoretical simulation of $\kappa_{i}$ vs. $E$-curves for $W_{i}=2$.

\subsubsection{Comparison between experimental and theoretically simulated plots of Li-ion conductivity as functions of the electrode potential}

Figure 2a-c shows respectively the theoretical plots of $C_{\text {int }}, D_{e i}$ and $\kappa_{i}$ as functions of the electrode potential for a number of values of the interaction parameter $g$; equations (33), (30) and (34) were used in this calculation $\left(W_{i}=1\right)$. The halfpeak width of the differential intercalation capacity peak strongly depends on the interaction parameter $g$, as was previously discussed $[4,23,24]$. At the critical value, $g_{\text {crit }}=-4$ (ideal first-order phase transition), the value of $C_{\text {int }}$ at the peak potential is infinitely high whereas $D_{e i} \rightarrow 0$. These features of the $C_{\text {int }}$ and $D_{e i}$ vs. $E$ plots manifest high intensity of the attractive interaction between the intercalation sites. It is interesting and practically significant that $\kappa_{i}$ passes at this potential through the same maximum as that for the $\kappa_{i}$ vs. $E$ curves calculated for $g>-4$ (monotonous charging of the electrode). The length of the two-phase co-existence region on the potential axis is about $7-8 \mathrm{mV}$ for $g=-5$ (see curve 5 in figure $2 \mathrm{a}-\mathrm{c}$ ). This region is centered at the related standard potential. Within the two-phase region $C_{\text {int }}$ takes formally negative values (figure 2a), whereas $D_{e i}$ traces a loop reaching a negative value of $D_{e i}$ at the standard potential (figure $2 \mathrm{~b}$ ). In contrast, although $\kappa_{i}$ shows also a loop in the vicinity of $E_{o}$, this loop is located near the invariable (with respect to $g$ ) real value for the peak of $\kappa_{i}$. This opens up the way for the most precise determination of the component diffusion coefficient $D_{i}^{\circ}$ since at $E=E_{o}$ the entropy factor $X(1-X)$ takes the maximum possible value 0.25 and all of the other numerical coefficients in equation (34) can be obtained experimentally.

In figure $3 \mathrm{a}-\mathrm{c}$ we present the same dependencies of $C_{\text {int }}, D_{e i}$ and $\kappa_{i}$ calculated with the use of equation (40), (42) and (32) for $W_{i}=2$ (the related intercalation isotherm is described by equation (39)). The related critical value of $g$ is $g_{\text {crit }}=-5.82$ instead of $g_{\text {crit }}=-4$ for the simple Frumkin isotherm (equation $(28), W_{i}=1$ ). Qualitatively, the curves in figure 3a-c reveal similar changes in their shape with $g$, as in the case of $W_{i}=1$ (see figure 2a-c); however, the shape of the corresponding curves is less symmetrical with respect to $E_{o}$ compared to rigorously symmetrical curves in figure 2a-c. An interesting consequence of this asymmetry in the shape is as follows: when $X$ approaches unity, the corresponding $D_{e i}$ takes values twice larger than that for $X=0$. This is an expected result since the limit of $D_{e i}$ at $X \rightarrow 1$ does 

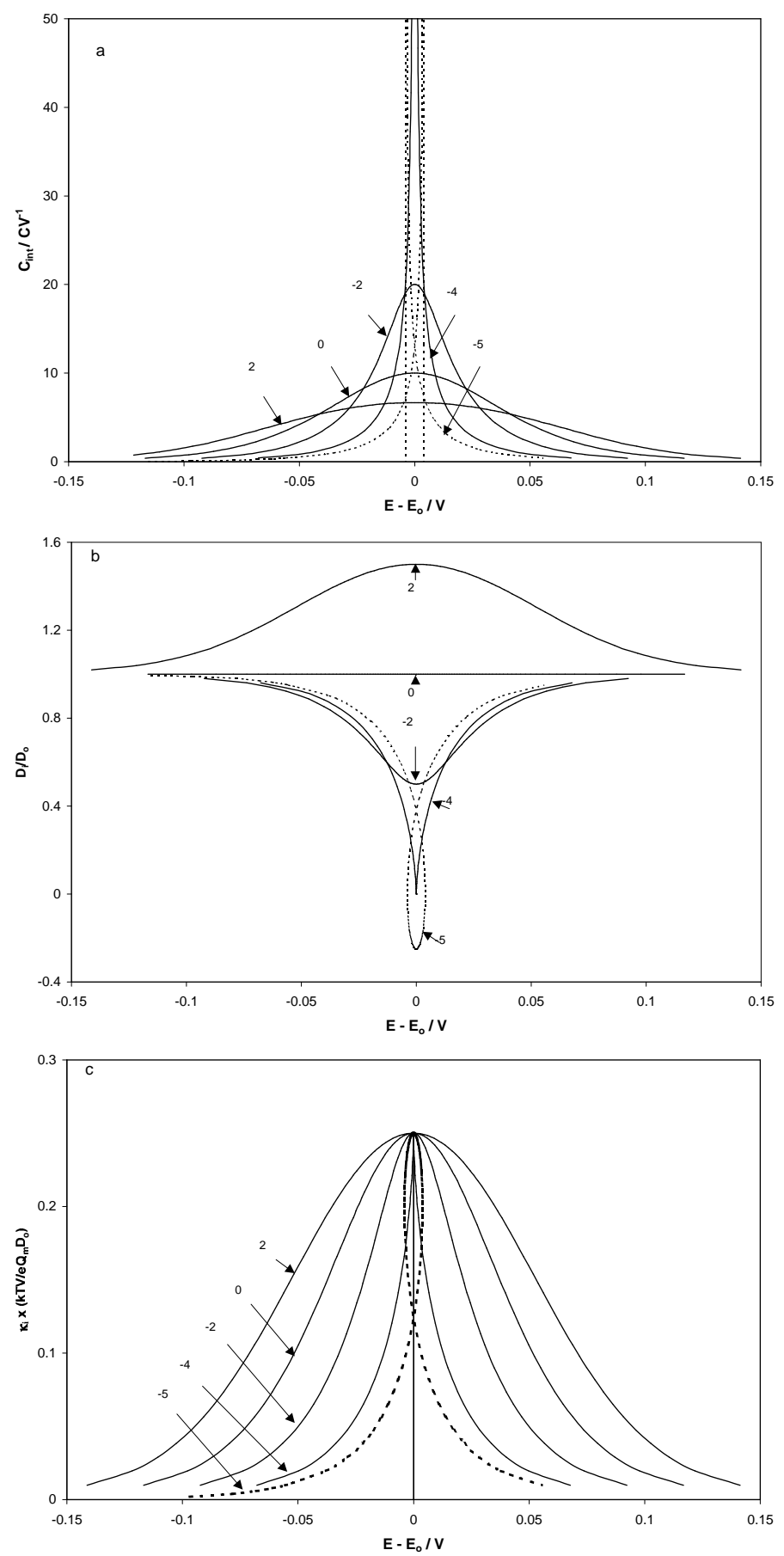

Figure 2. Potential dependence of theoretically simulated curves related to a simple Frumkin-type isotherm $\left(W_{i}=1\right)$ : the differential intercalation capacity, $C_{\text {int }}$ (a), equations (33) and (35); the dimensionless chemical diffusion coefficient, $D_{e i} / D_{i}$ (b), equations (30) and (35); and the dimensionless partial ionic conductivity $\kappa_{i}\left(k T V / e Q_{m} D_{i}^{\circ}\right)$ (c), equations (32) and (35). Numbers at curves denote the value of the interaction parameter $g$. 

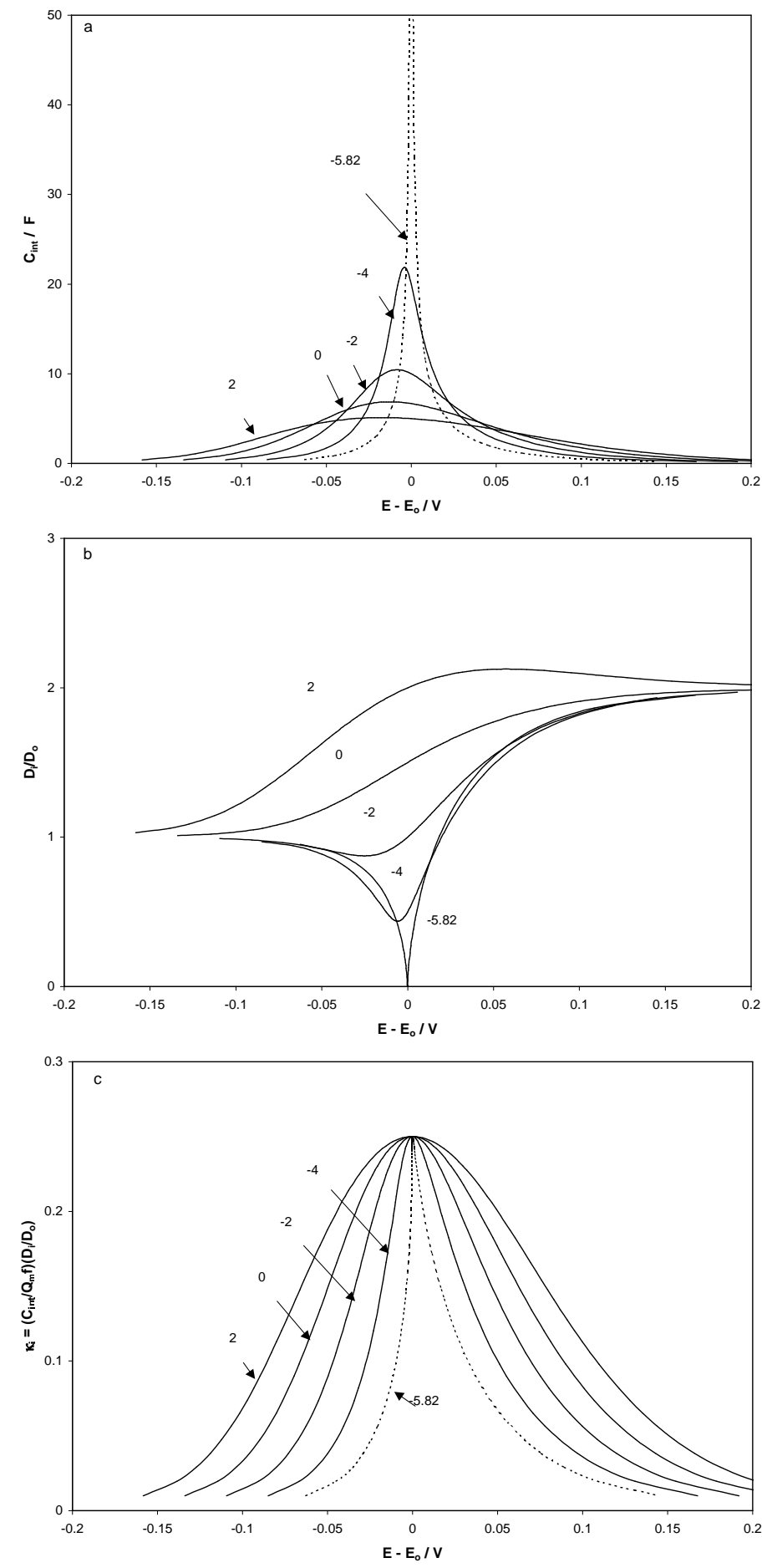

Figure 3. Potential dependence of theoretically simulated curves related to a modified isotherm for $W_{i}=2$, equation (39): the differential intercalation capacity, $C_{\text {int }}$ (a), equations (40) and (35); the dimensionless chemical diffusion coefficient. $D_{e i} / D_{i}^{\circ}$, (b), equations (42) and (35); and the dimensionless partial ionic conductivity $\kappa_{i}\left(k T V / e Q_{m} D_{i}^{\circ}\right)$ (c), equations (32) and (35). Numbers at curves denote the value of the interaction parameter $g$. 

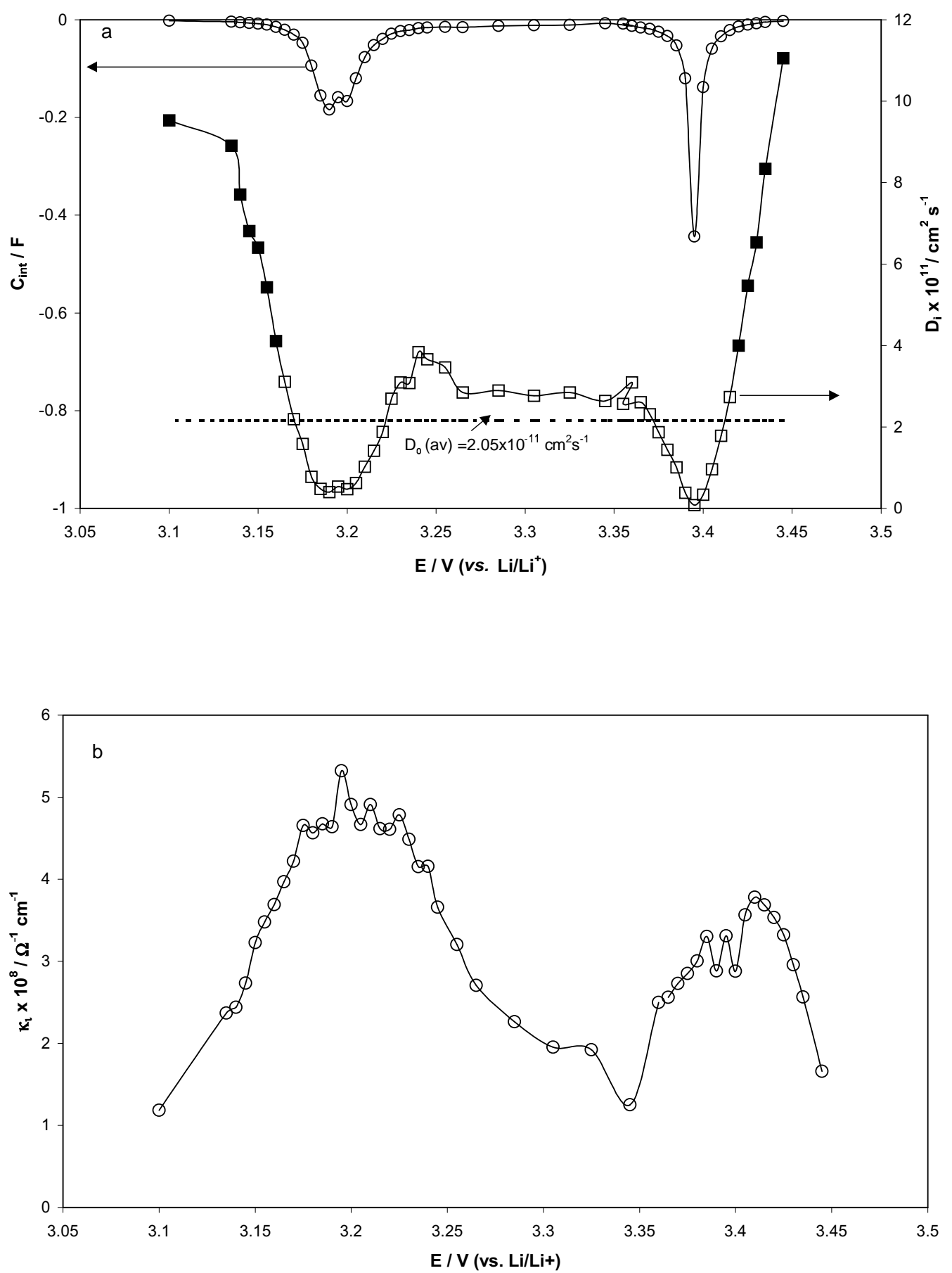

Figure 4. Potential dependencies of $C_{\text {int }}$ and $D_{e i}$ (a) and $\kappa_{i}$ (b) calculated for a vacuum-deposited $\mathrm{V}_{2} \mathrm{O}_{5}$ film $1600 \AA$ thick. Broken horizontal line relates to average value of $D_{i}^{\circ}$ obtained from the both peaks of $\kappa_{i}$. 
not depend any longer on the factor $(1-X)$ and approaches the theoretical limit $2 D_{e i}(X=0)$ (see equation $(42)$ ).

Figure 4 a-c shows the plots of $C_{\text {int }}, D_{e i}$ and $\kappa_{i}$ vs. potential obtained with $1600 \AA$ thick vacuum-deposited $\mathrm{V}_{2} \mathrm{O}_{5}$ film. The characteristic of this thin-film electrode, which does not contain conductive additives is the most appropriate for comparison with that simulated theoretically. All other experimental details related to PITT measurements have been previously reported [9]. $\kappa_{i}$ was calculated using equation (36), the characteristic diffusion length $l$ was assumed to coincide with film's thickness. Under these assumptions, the values of $\kappa_{i}$ ranged from $1 \cdot 10^{-8}$ to $5 \cdot 10^{-8} \Omega^{-1} \mathrm{~cm}^{-1}$. This range of $\kappa_{i}$ values is five orders of magnitude less than the specific electronic conductivity of the $\mathrm{V}_{2} \mathrm{O}_{5}$ film, $\kappa_{e}=1.6 \cdot 10^{-3} \Omega^{-1} \mathrm{~cm}^{-1}$ [8], thus $\kappa_{e} \gg \kappa_{i}$, and hence $t_{e}=1$.

Both two peaks on the $C_{\text {int }}$ and $D_{e i}$ vs. $E$ curves are suggested to be centered around 3.2 and $3.4 \mathrm{~V}$ (vs. $\mathrm{Li} / \mathrm{Li}^{+}$) related to first-order phase transitions. In fully delithiated state (small $X) \mathrm{V}_{2} \mathrm{O}_{5}$ consists of pure orthorhombic $\alpha$-phase, which is transformed to $\varepsilon$-phase via a first-order phase transition $(0.1<X<0.35$, the peak around $3.4 \mathrm{~V}$ (vs. $\left.\mathrm{Li} / \mathrm{Li}^{+}\right)$). The $\varepsilon$-phase undergoes only small structural changes in the range of $0.35<X<0.5$ (monotonous discharge in between the two phases). With further increase in $X, \varepsilon$-phase co-exists with a $\delta$-phase $(0.5<X<0.9)$ with the related peak around $3.2 \mathrm{~V}$ (vs. $\mathrm{Li} / \mathrm{Li}^{+}$). At $X=1$ the lithiated $\mathrm{V}_{2} \mathrm{O}_{5}$ presents almost pure $\delta$-phase.

If the actual attractive constant $g$ would be equal to the critical value $g_{\text {crit }}=-4$, then the half-peak widths of both $D_{e i}$ and $\kappa_{i}$ vs. $E$ curves should be as small as $11 \mathrm{mV}$ (see figure $2 \mathrm{~b}$ and $2 \mathrm{c}$ ). However, practically as follows from figure $4 \mathrm{a}$ and $4 \mathrm{~b}, D_{e i}$ and $\kappa_{i}$ vs. $E$ curves are much more broader as compared not only to the theoretical curves but also to the experimental $C_{\text {int }}$ vs. $E$ curves. This emphasizes once again (see also our previous discussion on the measurements of the chemical diffusion coefficient within two-phase co-existence regions $[13,24])$ that the related Cottrell slopes, $I t^{1 / 2}$ required for calculation of $\kappa_{i}$, equation (36), can be distorted due to an Ohmic potential drop or kinetic limitations (either interfacial or controlled by a nucleation step [27]). However, despite this complication, we made an attempt to evaluate the values of the component coefficient $D_{i}^{\circ}$ from the both two peaks at 3.4 and $3.2 \mathrm{~V}$ (vs. $\mathrm{Li} / \mathrm{Li}^{+}$) using equation (34). The values of $D_{i}^{\circ}$ obtained were close to each other: $1.9 \cdot 10^{-11}$ and $2.2 \cdot 10^{-11} \mathrm{~cm}^{2} / \mathrm{s}$, respectively. A broken horizontal line in figure $4 \mathrm{a}$ related to the average value of the component diffusion coefficient of Li-ion calculated from the both peaks: $D_{i}^{\circ}=2.05 \cdot 10^{-11} \mathrm{~cm}^{2} / \mathrm{s}$. As is seen from figure $4 \mathrm{a}$, this value is fully compatible with equation (30). In fact, the minima on the $D_{e i}$ vs. $E$ curve, related to two-phase co-existence regions (highly attractive interactions between the intercalation sites), are located below this line whereas the values of $D_{i}^{\circ}$ related to the single-phase region (continuous discharge) appear to be slightly above the line. In the framework of the simple lattice gas model presented above this indicates that in the single-phase region separating the two regions of two-phase co-existence, repulsion dominates among the interaction forces between the intercalation sites (equation (30) with $g>0$, compare with curve 2 in figure $2 \mathrm{~b}$ ). 
Later similar feature, but in much more pronounced form, will be reported and discussed for $\mathrm{Li}_{X} \mathrm{CoO}_{2}, \mathrm{Li}_{X} \mathrm{NiO}_{2}$ and $\mathrm{Li}_{X} \mathrm{Co}_{0.2} \mathrm{Ni}_{0.8} \mathrm{O}_{2}$ electrodes.

Here one important advantage of the proposed method of calculation of $D_{e i}$ should be emphasized. From figure $4 \mathrm{a}$ it is seen that at the very beginning of the Li-intercalation $\left(X<0.1, E>3.425 \mathrm{~V}\right.$ (vs. $\left.\mathrm{Li} / \mathrm{Li}^{+}\right)$) and at the end of the process $\left(X>0.9, E<3.155 \mathrm{~V}\right.$ (vs. $\left.\left.\mathrm{Li} / \mathrm{Li}^{+}\right)\right)$, the values of $D_{e i}$, shown as filled squares, appear to heavily deviate upwards from the horizontal line. These points relate to very small values of $C_{\text {int }}$ such that it is difficult to measure precisely the changes in $X$ and in the Cottrell slope $I t^{1 / 2}$ for the neighbouring potential increments (equations (32) and (36)). Practically, both these two quantities when related to a thin-film electrode containing a few milligram of the host material can be severely distorted by some parasitic background currents. The ratio between the incremental intercalation charge and the parasitic one is clearly increased in the vicinity of the $C_{\text {int }}$ maxima. Relative error when measuring $D_{e i}$ in the vicinity of the minima in figure 4 a did not exceed several percents However, for some extreme situations, within the region of two-phase co-existence, the typical Cottrell-like behaviour can be completely masked by some other than diffusion rate-determining steps. Such a behaviour has been recently reported for the Li-insertion-deinsertion in the vicinity of the $3 \mathrm{~V}$ plateau on the charging-discharging curves of $\mathrm{Li}_{X} \mathrm{Mn}_{2} \mathrm{O}_{4}$ spinel, which relates to slow droplet formation of a new phase in the bulk of the old one [27]. Another important conclusion resulting from figure $4 \mathrm{a}$ is that the determination of $D_{i}^{\circ}$ described above aids significantly to evaluate the reliability of the measured $D_{e i}$ for the two least precisely characterized regions related to either small $X$ or in the vicinity of unity.

Similar to thin-coated $\mathrm{V}_{2} \mathrm{O}_{5}$ electrode, we measured $\kappa_{i}$ vs. $E$ plots for a large number of composite carbonaceous anodes and transition metal cathodes. Figure 5a shows three main peaks on both $C_{\text {int }}$ and $\log D_{e i}$ vs. $E$ curves obtained with a composite graphite electrode [11]. From figure $5 \mathrm{~b}$ one can see the relevance of these peaks to the maxima on the $\kappa_{i}$ vs. $E$ curve. The values of $D_{i}^{\circ}$ for three major twophase co-existence regions were obtained similar to the case of the $\mathrm{V}_{2} \mathrm{O}_{5}$ electrode. $D_{i}^{\circ}$ was found to be $1.6 \cdot 10^{-9}, 1.0 \cdot 10^{-9}$ and $2.0 \cdot 10^{-9} \mathrm{~cm}^{2} / \mathrm{s}$ for the following two-phase coexistence regions: diluted phase I - phase IV (the most anodic peak), phase III - phase II (the middle peak) and phase II - phase I (the less positive peak). Thus the component diffusion coefficient of Li-ions appears to be constant within a factor of 2 for the whole range of $X$.

Figure 6a presents $C_{\text {int }}$ and $\log D_{e i}$ vs. $E$ curves for a disordered carbon electrode obtained from Mitsubishi. In contrast to graphite, a maximum in the $D$ vs. $E$ curve rather than a minimum is clearly seen in this case. Our model calculations predict the appearance of the maximum on the $D_{e i}$ vs. $E$ curves as a result of the effective repulsive interaction between the intercalation sites. We speculate that the specific disordered structure of this carbonaceous material promotes effectively repulsive interactions between the intercalation sites [12]. In the case of graphite, a minor contribution of repulsive interactions between the Li-ions on the neighbouring graphene planes is overcome by the highly attractive intra-layer interactions [6]. The lack of order in the disordered carbon obviously diminishes the role of the latter 

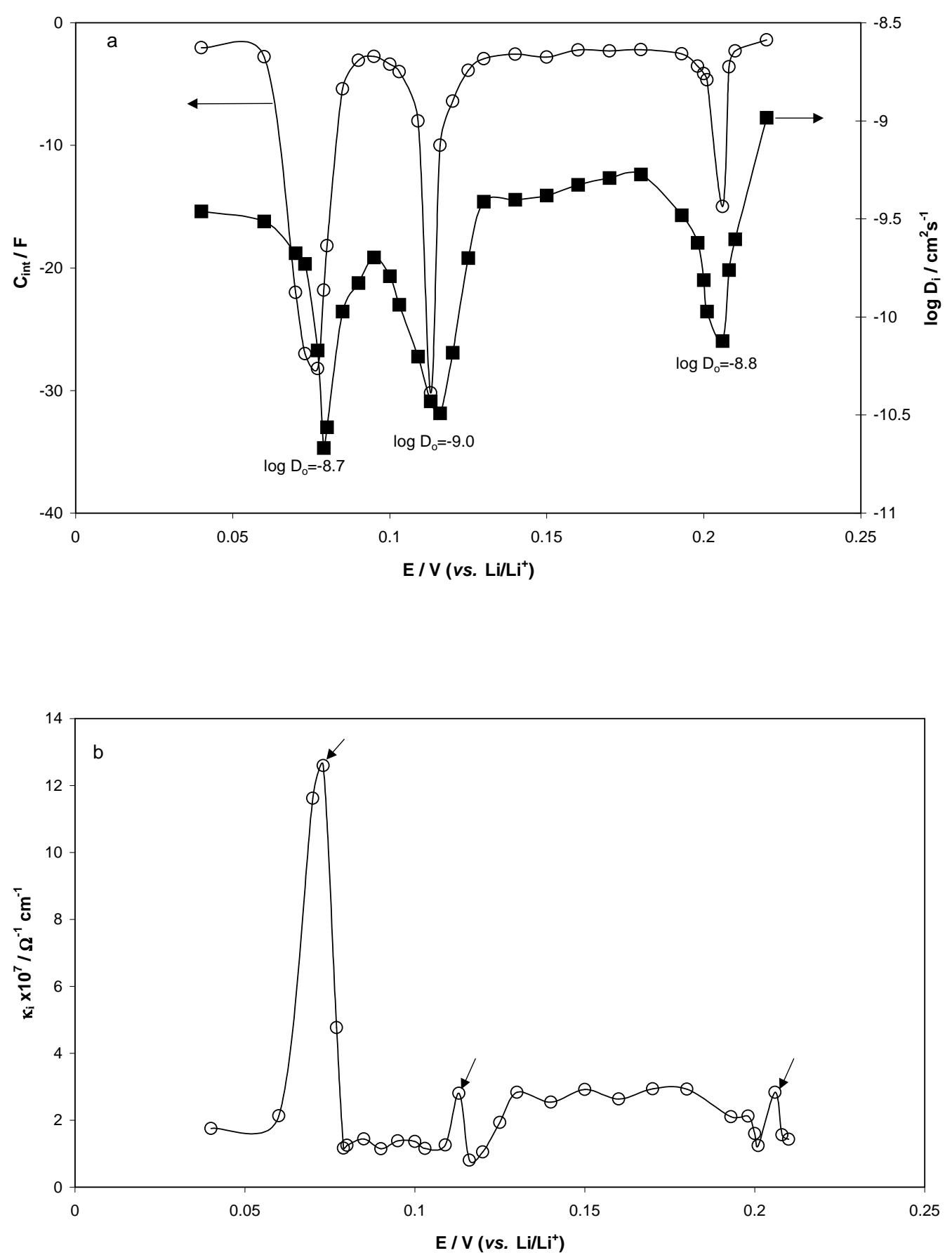

Figure 5. Potential dependencies of $C_{\mathrm{int}}$ and $D_{e i}$ (a) and $\kappa_{i}$ (b) calculated for a thin composite graphite electrode. 

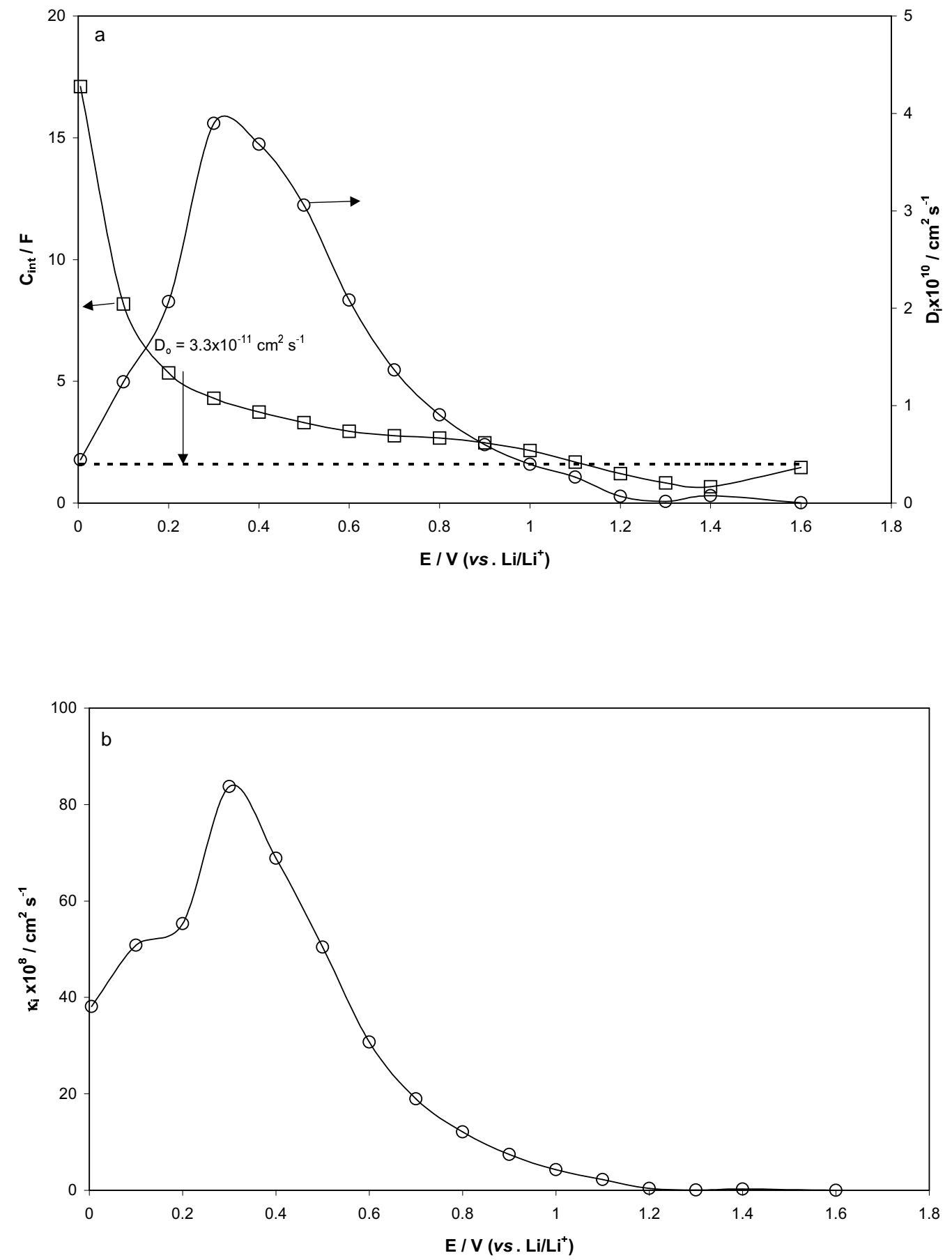

Figure 6. Potential dependencies of $C_{\text {int }}$ and $D_{e i}$ (a) and $\kappa_{i}$ (b) calculated for a thin composite disordered carbon electrode $N X-1$. 
factor. As is seen from figure $6 \mathrm{~b}, \kappa_{i}$ of the Li-ions in the disordered carbon varies in the range from $1 \cdot 10^{-8}$ to $8.4 \cdot 10^{-7} \Omega^{-1} \mathrm{~cm}^{-1}$. Using equations (34) and (36) we calculated $D_{i}^{\circ}$ from the value of $\kappa_{i}$ related to the maximum of the curve shown in figure $6 \mathrm{~b}$ : $D_{i}^{\circ}$ turned out to be equal to $3.3 \cdot 10^{-11} \mathrm{~cm}^{2} / \mathrm{s}$. This component diffusion coefficient is shown as a broken horizontal line in figure 6a. In agreement with the model proposed the measured chemical diffusion coefficient represents a bell-shaped curve, although not ideally symmetric, located above the horizontal line within the whole range of intercalation potentials (compare with figure 2 , curve with $g=2$ ).

Figure $7 \mathrm{a}$ and $b$ shows $C_{\text {int }}, \log D_{e i}$ and $\kappa_{i}$ vs. $E$ curves calculated for a thin powdery $\mathrm{Li}_{X} \mathrm{Mn}_{2} \mathrm{O}_{4}$ spinel electrode [27]. The data are qualitatively similar to that for the $\mathrm{V}_{2} \mathrm{O}_{5}$ electrode. Two intercalation steps of Li-insertion into the host lattice are manifested by two rather sharp peaks on the $C_{\text {int }}$ vs. $E$ curve and the corresponding minima on the $\log D_{e i}$ vs. $E$ curve. These steps reflect two first-order phase transitions in cubic spinel related to the ordered insertion of Li-ions into (8a) tetrahedral sites. Electronic conductivity of $\mathrm{LiMn}_{2} \mathrm{O}_{4}$ does not show any insulator-to-metal transition on delithiation, keeping values of the order of $\kappa_{e}=10^{-5} \Omega^{-1} \mathrm{~cm}^{-1}$ typical of semiconductors. Comparing this value with the values of the specific ionic conductivity of Li-ions in the range from $10^{-11}$ to $10^{-10} \Omega^{-1} \mathrm{~cm}^{-1}$ (see figure $7 \mathrm{~b}$ ), one can conclude that $t_{e}=1$ and that the mobility of electrons in the $\mathrm{Li}_{X} \mathrm{Mn}_{2} \mathrm{O}_{4}$ host is much higher than that of Li-ions. Two distinct and rather sharp maxima are seen on the $\kappa_{i}$ vs. $E$ curve of the $\mathrm{Li}_{X} \mathrm{Mn}_{2} \mathrm{O}_{4}$ electrode, related to the peaks on the $C_{\text {int }}$ and $\log D_{e i}$ vs. $E$ curves. The component diffusion coefficient $D_{i}^{\circ}$ calculated from the peaks on the $\kappa_{i}$ vs. $E$ curve was close to $1.3 \cdot 10^{-11} \mathrm{~cm}^{2} / \mathrm{s}\left(\log D_{i}^{\circ}=-10.9\right)$. This value is compatible with equations (30) and (31): as is seen from figure 7a, the values of $D_{e i}$ drastically decrease within the range of potentials related to the peaks of $C_{\text {int }}\left(W_{i}^{\circ}<1\right)$. Somewhat higher values of $D_{e i}$ compared to $D_{i}^{\circ}$ in the range of potentials from 3.75 to $3.90 \mathrm{~V}$ (vs. $\mathrm{Li} / \mathrm{Li}^{+}$) are probably caused by the decrease in precision of the measured PITT data: they relate to small changes in the $C_{\text {int }}$ and the Cottrell slopes $I t^{1 / 2}$ (i.e. at $X \ll 1$ and in the vicinity of $X=1$ ).

Figure $8 \mathrm{a}$ and b shows the potential dependencies of $C_{\text {int }}, \log D_{e i}[13]$ and $\kappa_{i}$ for a composite $\mathrm{Li}_{X} \mathrm{CoO}_{2}$ electrode, which is of great interest in view of the insulator-tometal transition occurring after partial Li-ion depletion from the electrode ( $X$ decreases from 1 to 0.75 ) [13]). The related potential range corresponds to co-existence of two hexagonal phases, followed by a single-phase region as $X$ increases to 0.5 [28]. We estimated the change in the specific electronic conductivity $\kappa_{e}$ from the total measured electronic conductivity related to the potential range from 3.0 to $4.3 \mathrm{~V}$ (vs. $\mathrm{Li} / \mathrm{Li}^{+}$): $\kappa_{e}$ increases from $10^{-10}$ to $4 \cdot 10^{-6} \Omega^{-1} \mathrm{~cm}^{-1}$. This increase occurs at the very beginning of the Li-deintercalation, thus in the vicinity of the peak in $C_{\text {int }}$ vs. $E$ curve $\kappa_{e}=4 \cdot 10^{-6} \Omega^{-1} \mathrm{~cm}^{-1}$ is much larger than the maximum value of $\kappa_{i}=10^{-8} \Omega^{-1} \mathrm{~cm}^{-1}$ (see figure $8 \mathrm{~b}$ ). Hence in this potential range $t_{e}=1$. The component diffusion coefficient $D_{i}^{\circ}$ was estimated as described above; it turns out to be $D_{i}^{\circ}=2.8 \cdot 10^{-11} \mathrm{~cm}^{2} / \mathrm{s}$. It is interesting that within the single-phase region, i.e. from ca. 3.95 to $4.15 \mathrm{~V}$ (vs. $\mathrm{Li} / \mathrm{Li}^{+}$) the specific ionic conductivity $\kappa_{i}$ increases once again (see figure 8b), but in this case the peak of $\kappa_{i}$ is rather broad. On the 

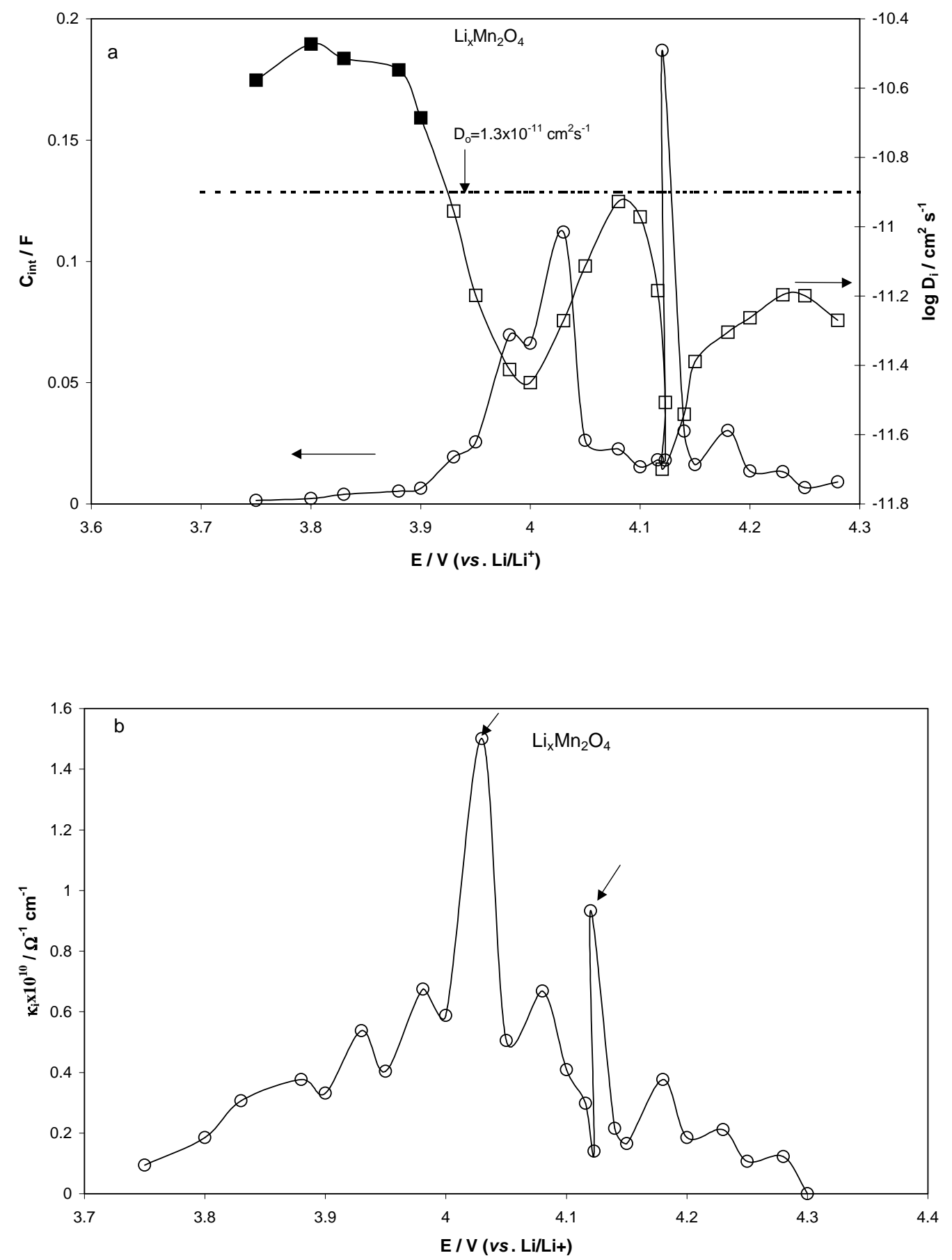

Figure 7. Potential dependencies of $C_{\text {int }}$ and $D_{e i}(\mathrm{a})$ and $\kappa_{i}$ (b) calculated for a thin composite $\mathrm{Li}_{X} \mathrm{Mn}_{2} \mathrm{O}_{4}$ spinel electrode. 

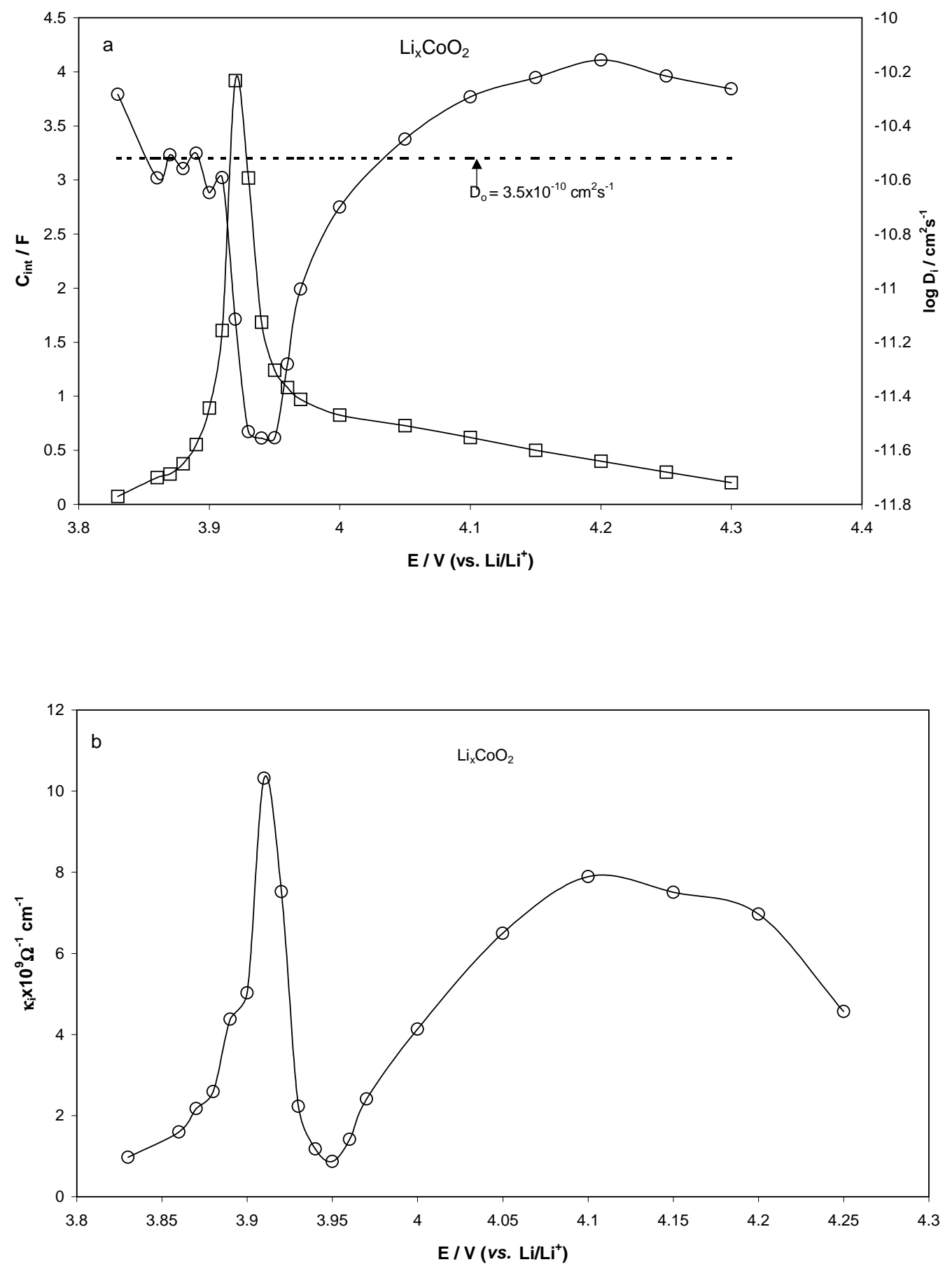

Figure 8. Potential dependencies of $C_{\mathrm{int}}$ and $D_{e i}$ (a) and $\kappa_{i}$ (b) calculated for a thin composite $\mathrm{Li}_{X} \mathrm{CoO}_{2}$ electrode. 
other hand, the dependence of $C_{\mathrm{int}}$ on the potential in this region is also rather flat whereas $\log D_{e i}$ tends to pass through a broad maximum in the vicinity of $E=$ $4.2 \mathrm{~V}$ (vs. $\mathrm{Li} / \mathrm{Li}^{+}$), see figure 8 a. Such behaviour of $\kappa_{i}, C_{\text {int }}$ and $D_{e i}$ is compatible with the positive value of the interaction constant $g$; this particular feature of the $\mathrm{Li}_{X} \mathrm{CoO}_{2}$ electrode qualitatively resembles the shape of the curve with $g=2$ in figure 2a-c. Note a very remarkable feature in figure 8a: the broken line corresponding to $D_{i}^{\circ}=2.8 \cdot 10^{-11} \mathrm{~cm}^{2} / \mathrm{s}$ marks the boundary, which separates the region of the two-phase co-existence with the minimum in $\log D_{e i}$ (the region from 3.8 to $4.0 \mathrm{~V}$ (vs. $\mathrm{Li} / \mathrm{Li}^{+}$) from the single-phase region in the potential range from 4.0 to $4.3 \mathrm{~V}$ (vs. $\mathrm{Li} / \mathrm{Li}^{+}$). This single-phase region relates to the effective repulsive interactions with a flat minimum on the $\log D_{e i}$ vs. $E$ curve centered at ca. $4.2 \mathrm{~V}$ (vs. $\mathrm{Li} / \mathrm{Li}^{+}$). The trend to exhibit effective repulsive interactions between the intercalation sites around $X=0.5$ becomes even more pronounced when turning from $\mathrm{Li}_{X} \mathrm{CoO}_{2}$ to $\mathrm{Li}_{X} \mathrm{NiO}_{2}$ and $\mathrm{Li}_{X} \mathrm{Co}_{0.2} \mathrm{Ni}_{0.8} \mathrm{O}_{2}$ electrodes.

The data related to the latter two electrodes are presented in figure $9 \mathrm{a}$ and $9 \mathrm{~b}$ and figure 10a and 10b, respectively. We have previously reported [29] that this pair of electrodes may serve as an example of a clear correlation between their crystallographic structure and the electrochemical behaviour. In fact, considering the whole range of intercalation potentials from 3.2 to $4.1-4.2 \mathrm{~V}$ (vs. $\mathrm{Li} / \mathrm{Li}^{+}$), in which $X$ decreases from unity to ca. $0.3-0.4$, we note that $C_{\text {int }}$ vs. $E$ curve for the $\mathrm{Li}_{X} \mathrm{Co}_{0.2} \mathrm{Ni}_{0.8} \mathrm{O}_{2}$ electrode is generally similar to the smoothed curve for the $\mathrm{Li}_{X} \mathrm{NiO}_{2}$ electrode (compare figures 10a and 9a, respectively). The curve related to the former figure contains peaks, which can be regarded as heavily flattened peaks of the $\mathrm{Li}_{X} \mathrm{NiO}_{2}$ electrode. Such a behaviour is in complete correspondence with the sequence of first-order phase transition occurring during Li-deintercalation from $\mathrm{Li}_{X} \mathrm{NiO}_{2}$ and with essentially single-phase reaction typical for $\mathrm{Li}_{X} \mathrm{Co}_{0.2} \mathrm{Ni}_{0.8} \mathrm{O}_{2}$. On the phase diagrams shown on the bottoms of figures $9 \mathrm{a}$ and $10 \mathrm{a} H_{1}$ and $H_{2}$ denote two different hexagonal phases whereas $M$ stands for the corresponding monoclinic phase (for details see [29]). In strict correspondence with rather sharp peaks on the $C_{\text {int }}$ vs. $E$ curve, deep minima on the $\log D_{e i}$ vs. $E$ curves represent a characteristic feature of the $\mathrm{Li}_{X} \mathrm{NiO}_{2}$ electrode. Changes in the $C_{\text {int }}$ and $\log D_{e i}$ vs. $E$ curves related to the $\mathrm{Li}_{X} \mathrm{Co}_{0.2} \mathrm{Ni}_{0.8} \mathrm{O}_{2}$ electrode are much smoother compared to that for the $\mathrm{Li}_{X} \mathrm{NiO}_{2}$ electrode, which is in accordance with the single-phase nature of the Li-deintercalation reaction for the former electrode.

The above mentioned specific difference in the electroanalytical behaviour of $\mathrm{Li}_{X} \mathrm{NiO}_{2}$ and $\mathrm{Li}_{X} \mathrm{Co}_{0.2} \mathrm{Ni}_{0.8} \mathrm{O}_{2}$ electrode is also clearly reflected in the corresponding $\kappa_{i}$ vs. $E$ plots. A rather broad minimum in $\kappa_{i}$ for the $\mathrm{Li}_{X} \mathrm{Co}_{0.2} \mathrm{Ni}_{0.8} \mathrm{O}_{2}$ electrode around $E=3.85 \mathrm{~V}$ (vs. $\mathrm{Li} / \mathrm{Li}^{+}$) looks like a heavily smoothed $\kappa_{i}$ vs. $E$ curve for the $\mathrm{Li}_{X} \mathrm{NiO}_{2}$ electrode (compare figures $9 \mathrm{~b}$ and $10 \mathrm{~b}$ ). The local peaks on the latter curve correspond well with the peaks on the related $C_{\text {int }}$ vs. $E$ curve.

Similar to all the above considered Li-intercalation electrodes, we calculated the values of $D_{i}^{\circ}$ in the maxima of $\kappa_{i}, E$ curves (see figures $9 \mathrm{~b}$ and $10 \mathrm{~b}$ ), which turned out to be $2.1 \cdot 10^{-12}$ and $1.6 \cdot 10^{-12} \mathrm{~cm}^{2} / \mathrm{s}$ for $\mathrm{Li}_{X} \mathrm{NiO}_{2}$ and $\mathrm{Li}_{X} \mathrm{Co}_{0.2} \mathrm{Ni}_{0.8} \mathrm{O}_{2}$, respectively. Logarithms of $D_{i}^{\circ}$ are shown in figures $9 \mathrm{a}$ and 10a as horizontal broken lines. Once 

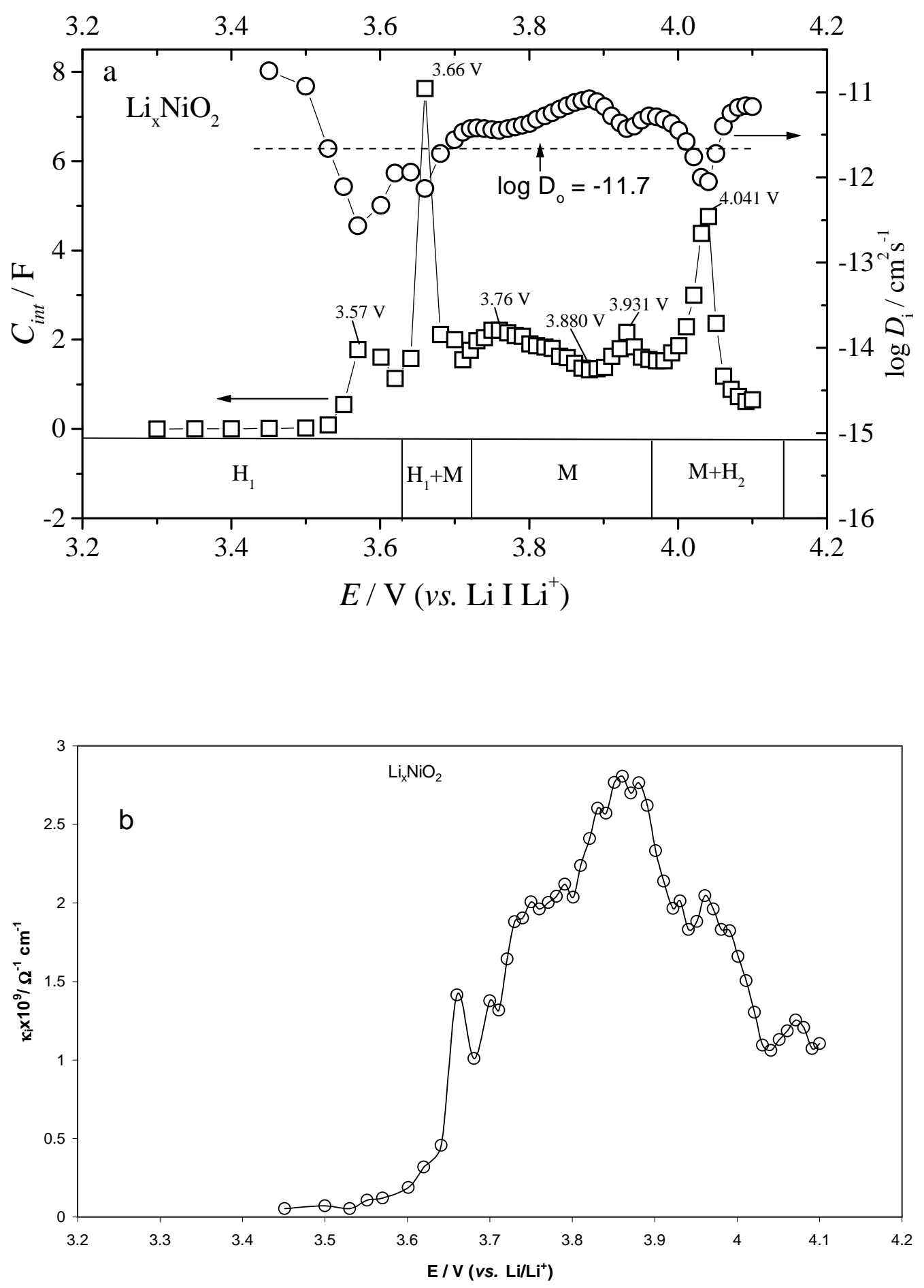

Figure 9. Potential dependencies of $C_{\mathrm{int}}$ and $D_{e i}$ (a) and $\kappa_{i}$ (b) calculated for a thin composite $\mathrm{Li}_{X} \mathrm{NiO}_{2}$ electrode. 

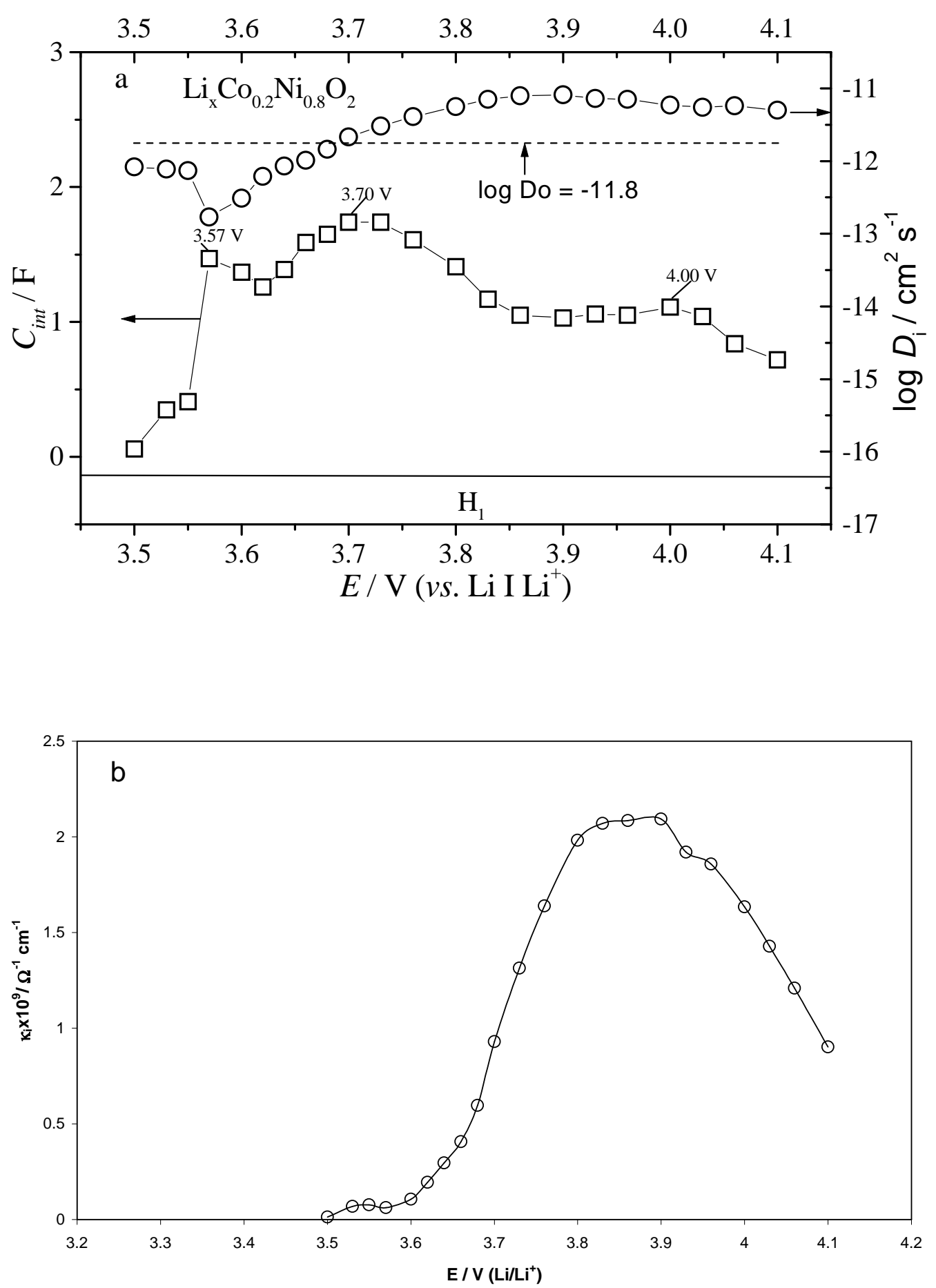

Figure 10. Potential dependencies of $C_{\text {int }}$ and $D_{e i}$ (a) and $\kappa_{i}$ (b) calculated for a thin composite $\mathrm{Li}_{X} \mathrm{Co}_{0.2} \mathrm{Ni}_{0.8} \mathrm{O}_{2}$ electrode. 
again, as in the case of $\mathrm{Li}_{X} \mathrm{CoO}_{2}$ electrode, these horizontal lines separate two-phase co-existence regions or the region with essentially high attractive interaction between the intercalation sites (the minima down from this line) from the single-phase region with the dominating repulsive interactions between the intercalation sites. Certainly, this is a remarkable and expected result in the framework of the proposed model (compare with figure $2 \mathrm{~b}$ ). Moreover, the broad peaks on the $\kappa_{i}$ vs. $E$ curves for the $\mathrm{Li}_{X} \mathrm{NiO}_{2}$ and $\mathrm{Li}_{X} \mathrm{Co}_{0.2} \mathrm{Ni}_{0.8} \mathrm{O}_{2}$ electrodes centered at ca. $3.85 \mathrm{~V}$ (vs. $\mathrm{Li} / \mathrm{Li}^{+}$) seem to be in excellent agreement with the potential dependence of the Li-ion charge transfer resistance, $R_{\mathrm{ct}}$ for the same electrodes measured using EIS [29]. In fact, we observed a rather sharp minimum on the charge-transfer resistance vs. potential curve at about 3.80-3.85 V (vs. $\mathrm{Li} / \mathrm{Li}^{+}$). This agreement is not of course an accidental one since we have shown that $R_{\mathrm{ct}}^{-1} \sim X(1-X)$ [29]. Thus $R_{\mathrm{ct}}^{-1}$ and, hence the corresponding ionic exchange current density are proportional to the product $X(1-X)$, as is the case of $\kappa_{i}$ (compare with equation (34)). This correlation (proportionality) between $i_{o}$ and $\kappa_{i}$ can be easily appreciated: both kinetic quantities, $i_{o}$ and $\kappa_{i}$ depend not only on the concentration of the reacting (or diffusing, as in the case of $\kappa_{i}$ ) species but also on the concentration of the unoccupied sides. In the case of $\kappa_{i}$ this is true because the species move (by hopping) from occupied to unoccupied sites. As related to the exchange current density, it reaches the maximum value at comparable concentrations of the both reacting species (here, occupied and unoccupied sites); at any deviation from the standard potential, corresponding to the maximum value of the factor $X(1-X)$, the supply of either reagent is insufficient to support a high reaction rate.

In conclusion, table 1 summarizes the values of the component diffusion coefficient $D_{i}^{\circ}$ of a large variety of the Li-insertion anodes and cathodes obtained from the maximum values of the partial Li-ion conductivity, which was measured using one and the same incremental technique (here, PITT). Since two characteristic lengths are involved in the calculation, the diffusion length and the thickness of the electrode, which do not necessarily coincide for the porous composite electrodes, we believe that these values can be compared with a precision better than one order of magnitude. It is seen from the table that $D_{i}^{\circ}$ for the Li-ions in graphite has the largest value whereas for the rest of the materials studied $D_{i}^{\circ}$ varies in the range between $10^{-11}$ and $10^{-12} \mathrm{~cm}^{2} / \mathrm{s}$.

\section{Conclusions}

The classical "enhancement factor" $W_{i}$, characterizing the ratio of the chemical and component diffusion coefficients, $D_{e i} / D_{i}$, for mixed-conduction electrodes in "dilute solution" approximation was compared with the new "enhancement factor" $W_{i}^{\circ}$, related to the same ratio but derived for a simple lattice-gas model approximation with interactions between the intercalation sites. The classical "enhancement factor" $W_{i}$ introduced by Weppner and Huggins depends on the ratios of the concentrations and mobilities of all electronic and ionic species participating in the mass-transport through a mixed-conduction electrode. The concept of $W_{i}$ is espe- 
Table 1. Component diffusion coefficient $D_{i}^{\circ}\left(\mathrm{cm}^{2} / \mathrm{s}\right)$ for a variety of Li-insertion electrodes determined by PITT, equations (14) and (36).

\begin{tabular}{|l|r|}
\hline Electrode & $D_{i}^{\circ}\left(\mathrm{cm}^{2} / \mathrm{s}\right)$ \\
\hline Synthetic graphite KS-6 & $1.0-2.0 \cdot 10^{-9}$ \\
\hline Disordered carbon NX-1 & $3.3 \cdot 10^{-11}$ \\
\hline $\mathrm{Li}_{X} \mathrm{~V}_{2} \mathrm{O}_{5}$ (vacuum-deposited film) & $1.9-2.2 \cdot 10^{-9}$ \\
\hline $\mathrm{Li}_{X} \mathrm{Mn}_{2} \mathrm{O}_{4}$ (composite) & $1.3 \cdot 10^{-11}$ \\
\hline $\mathrm{Li}_{X} \mathrm{CoO}_{2}$ (composite) & $2.8 \cdot 10^{-11}$ \\
\hline $\mathrm{Li}_{X} \mathrm{NiO}_{2}$ (composite) & $2.1 \cdot 10^{-11}$ \\
\hline $\mathrm{Li}_{X} \mathrm{Co}_{0.2} \mathrm{Ni}_{0.8} \mathrm{O}_{2}$ (composite) & $1.6 \cdot 10^{-12}$ \\
\hline
\end{tabular}

cially useful for determination of thermodynamic and kinetic properties of various Li-alloys. We have extended the classical approach to a more general model, which implies the connection of the new "enhancement factor" $W_{i}^{\circ}$ with a family of intercalation isotherms of the Nernstian and non-Nernstian type, depending on the stoichiometry of the association reaction between the electronic and ionic species in the electrode bulk. $W_{i}^{\circ}$ reflects quite a different property, as compared to that of $W_{i}$, namely, the effect of interactions between the intercalated sites on the chemical diffusion coefficient of Li-ions. Thus $W_{i}^{\circ}$ describes the deviation of the intercalation isotherm from the Langmurian one.

In the case of "dilute solution" approximation the partial ionic conductivity is parameterized by a quantity proportional to the product of the ionic electric mobility (and, hence, the component diffusion coefficient $\mathrm{D}_{i}$ ) and the concentration. In contrast, the partial ionic conductivity $\kappa_{i}$ within the lattice-gas approach was shown to be parameterized by a quantity proportional to the product of the chemical diffusion coefficient $D_{e i}$ and the differential intercalation capacity $C_{\text {int }}$. Experimentally, $\kappa_{i}$ can be determined as a function of potential using one of the available smallamplitude technique, e.g. PITT. Comparing the height of the peaks on the experimental $\kappa_{i}$ vs. $E$ curves with the theoretical equation, we have proposed a simple and reliable method of determination of the component diffusion coefficient $D_{i}^{\circ}$. Using 7 different intercalation electrodes (synthetic graphite, disordered carbon, $\mathrm{Li}_{X} \mathrm{~V}_{2} \mathrm{O}_{5}$, $\mathrm{Li}_{X} \mathrm{Mn}_{2} \mathrm{O}_{4}, \mathrm{Li}_{X} \mathrm{CoO}_{2}, \mathrm{Li}_{X} \mathrm{NiO}_{2}$ and $\left.\mathrm{Li}_{X} \mathrm{Co}_{0.2} \mathrm{Ni}_{0.8} \mathrm{O}_{2}\right)$ we were able to discriminate between the potential regions, in which either attractive or repulsive interactions between the intercalation sites play the dominant role. In addition, the proposed method aids in recognizing the unreliable points on the $D_{e i}$ vs. $E$ curve related to the very beginning and the end of the intercalation process. We have found a direct correlation of the shape of the $\kappa_{i}$ vs. $X$ curve with the dependence of the ion-exchange current density vs. $X$. This correspondence is based on the common entropy factor $X(1-X)$ influencing the both above quantity. Of principle importance is the correlation between the differential intercalation capacity curves, $C_{\text {int }}$ 
vs. $E$ and the partial Li-ion conductivity vs. potential curves (both are bell-shaped curves), which has been observed for all 7 intercalation electrodes under consideration. This correlation means that the differential intercalation capacity is controlled by availability of sites for the Li-ion insertion and not by availability of sites for insertion of charge counter-balancing electronic species. This conclusion is in agreement with the reported relatively high partial electronic conductivity of the most of Li-insertion electrodes under consideration.

\section{References}

1. Weppner W., Huggins R.A. // J. Electrochem. Soc., 1977, vol. 124, p. 1569.

2. Wen C.J., Boukamp B.A., Huggins R.A., Weppner W. // J. Electrochem. Soc., 1979, vol. 126 , p. 2258.

3. Weppner W., Huggins R.A. // Ann. Rev. Mater. Sci., 1978, p. 1569.

4. Chidsey C.E.D., Murray R.W. // J. Phys. Chem., 1986, vol. 90, p. 1479.

5. Coleman S.T., McKinnon W.R., Dahn J.R. // Phys. Rev. B, 1984, vol. 29, p. 4147.

6. McKinnon W.R., Haering R.R. Modern Aspects in Electrochemistry. Vol. 15. NY, Plenum Press, 1987, p. 235.

7. Ho C., Raistrick I.D., Huggins R.A. // J. Electrochem. Soc., 1980, vol. 127, p. 343.

8. Levi M.D., Lu Z., Gofer Y., Cohen Yaron, Cohen Yair, Vieil E., Serose J., Aurbach D. // J. Electroanal. Chem., 1999, vol. 479, p. 12.

9. Levi M.D., Lu Z., Aurbach D. // Solid State Ionics, 2001, vol. 143, p. 309.

10. Levi M.D., Aurbach D. // J. Phys. Chem. B, 1997, vol. 101, p. 4630.

11. Levi M.D., Aurbach D. // J. Phys. Chem. B, 1997, vol. 101, p. 4641.

12. Gnanaraj J.S., Levi M.D., Levi E., Salitra G., Aurbach D., Fischer J.E., Claye A. // J. Electrochem. Soc., 2001, vol. 148, p. A525.

13. Levi M.D., Salitra G., Markovsky B., Teller H., Aurbach D. // J. Electrochem. Soc., 1999, vol. 146, p. 1279.

14. Aurbach D., Levi M.D., Levi E., Teller H., Markovsky B., Salitra G, Heider U., Heider L. // J. Electrochem. Soc., 1998, vol. 145, p. 3024.

15. Levi M.D., Gamolsky K., Heider U., Oesten R., Aurbach D. // J. Electroanal. Chem., 1999, vol. 477, p. 32.

16. Aurbach D. // J. Electrochem. Soc., 1989, vol. 136, p. 906, p. 1606, p. 1611.

17. Aurbach D., Gottlieb H.E. // Electrochim. Acta, 1989, vol. 34, p. 141.

18. Newman J.S. Electrochemical Systems. $2^{\text {nd }}$ ed. NJ, Prentice Hall, Englewood Cliffs, 1991.

19. Vorotyntsev M.A., Badiali J.P., Vieil E. // Electrochim. Acta, 1996, vol. 41, p. 1375.

20. Vorotyntsev M.A., Kornyshev A.A. // Rep. Acad. Sci. USSR, 1976, vol. 230, p. 631.

21. Kornyshev A.A., Vorotyntsev M.A. // Electrochim. Acta, 1978, vol. 23, p. 267.

22. Kornyshev A.A., Vorotyntsev M.A. // Electrochim. Acta, 1981, vol. 26, p. 303.

23. Vorotyntsev M.A., Badiali J.P. // Electrochim. Acta, 1994, vol. 39, p. 289.

24. Levi M.D., Aurbach D. // Electrochim. Acta, 1999, vol. 45, p. 167.

25. Vorotyntsev M.A., Daikhin L.I., Levi M.D. // J. Electroanal. Chem., 1992, vol. 332, p. 213.

26. Tsirlina G.A., Petrii O.A., Levi M.D., Aurbach D. // Electrochim. Acta, 2001, vol. 46, p. 4141. 
27. Levi M.D., Gamolsky K., Aurbach D., Heider U., Oesten R. // J. Electrochem. Soc., 2000, vol. 147, p. 25.

28. Reimers J.N., Dahn J.R. // J. Electrochem. Soc., 1992, vol. 138, p. 2091.

29. Levi M.D., Gamolsky K., Heider U., Oesten R., Aurbach D. // Electrochim. Acta, 2000, vol. 45, p. 1781.

\title{
Інтерпретація експериментів з застосуванням техніки з переривистим потенціалом для різних літієво-інтеркаляційних електродів
}

\author{
М.Д.Леві ${ }^{1}$, Д.Аурбах ${ }^{1}$, М.А.Воротинцев ${ }^{2}$
}

1 Хімічний факультет, Університет Бар-Ілан, Рамат-Ґан 52900, Ізраїль

2 Факультет природничих наук, Бурґундський уніветситет, бульв. Габріель 6, 21000 Діжон, Франція

Отримано 15 листопада 2001 р.

В цій роботі ми порівнюємо два різних підходи для розрахунку підсилюючого фактора $W_{i}$, ґрунтуючись на його означенні як співвідношенні коефіцієнтів хімічної та компонентної дифузії для компонент в електродах із змішаною провідністю. Ці підходи беруть свій початок від моделей "розведеного розчину" або “ґраткового газу" для іонних систем. Перший з цих підходів застосовний лише у випадку малих змін іонної концентрації, тоді як другий дозволяє розглядати інтеркаляції у широкому діапазоні рівнів. Коефіцієнт компонентної дифузії іонів літію був визначений для серії анодів і катодів інтеркальованих літієм. Був означений новий “підсилюючий фактор" для іонного транспорту та встановлений його зв'язок з інтеркаляційною теплоємністю та ізотермою інтеркаляції. Спостерігалась кореляція між залежностями диференціальної ємності від потенціалу та парціальної іонної провідності від потенціалу. Це розглядається як доказ того, що інтеркаляційний процес контролюється доступністю центрів для розміщення іонів $\mathrm{Li}$, а не одночасним розміщенням збалансовуючих електронних компонент.

Ключові слова: літієво-іонні батареї, бінарні компоненти, коефіцієнти хімічної дифузії, іонна провідність, модель граткового газу

PACS: $84.60 . D n, 66.30 . D n$ 